\title{
Electric Field-Mediated Fibronectin-Hydroxyapatite Interaction: A Molecular Insight
}

\author{
Subhadip Basu, Biswajit Gorai, Bikramjit Basu,* and Prabal K. Maiti* \\ Cite This: J. Phys. Chem. B 2021, 125, 3-16 \\ Read Online
}

ABSTRACT: In experimental research-driven biomaterials science, the influence of different material properties (elastic stiffness, surface energy, etc.) and, to a relatively lesser extent, biophysical stimulation (electric/magnetic) on cell-material interactions has been extensively investigated. Despite the central importance of protein adsorption on cell-material interactions, the quantitative analysis to probe into the role of physicochemical factors in protein adsorption remains largely unexplored in biomaterials science. In recent studies, the critical role of electric field stimulation toward the modulation of cell functionality in implantable biomaterials has been experimentally demonstrated. Given this background, we investigated the influence of external electric field stimulation (upto $1.00 \mathrm{~V} / \mathrm{nm}$ ) on fibronectin (FN) adsorption on a hydroxyapatite (HA) (001) surface at $300 \mathrm{~K}$ using the
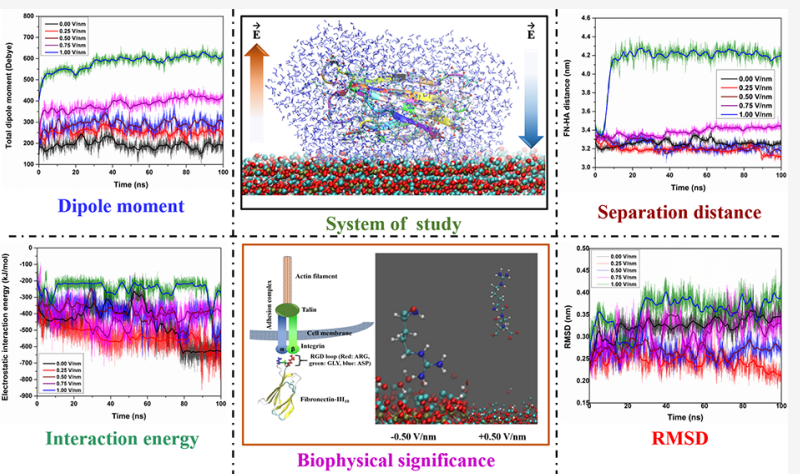
all-atom molecular dynamics (MD) simulation method. FN adsorption was found to be governed by attractive electrostatic interactions, which changed with the electric field strength. Nonmonotonous changes in the structural integrity of FN were recorded with the change in the field strength and direction. This can be attributed to the spatial rearrangement of the positions of local charges and the global structural changes of proteins. The dipole moment vectors of FN, water, and HA quantitatively exhibited a similar pattern of orienting themselves parallel to the field direction, with field strength-dependent increase in their magnitudes. No significant change has been recorded for the radial distribution function of water surrounding FN. Field-dependent variation in the salt bridge nets and the number of hydrogen bonds between FN and HA were also examined. One of the important results in the context of cell-material interaction is that the RGD (Arg-Gly-Asp) sequence of FN was exposed to the solvent side when the field was applied along an outward direction perpendicular to the HA (001) surface. In summary, the present study provides molecular insights into the influence of electric field stimulation on phenomenological interactions involved in FN adsorption on the HA surface.

\section{INTRODUCTION}

A number of unmet clinical needs have triggered significant research activities to develop new biomaterials and bioengineering strategies. The quantitative understanding of the nature of interactions between material surfaces and the components of living systems (proteins, cells, bacteria, blood, etc.) is of utmost significance for rational designing of new biomaterials. The very first step of this interaction is the adsorption of proteins on the biomaterial surface. When a biomaterial is implanted in a human subject, the abundance of adsorbed proteins on the material surface initiates a cascade of biophysical events like platelet activation and foreign body response. ${ }^{1}$ It is the adsorbed proteins that interact with cell surface receptors to result in the formation of focal adhesion complexes. ${ }^{2,3}$ Therefore, the cellmaterial interaction (interplay between biological cells and inorganic surfaces, mediated by the adsorbed proteins on the surface) and cellular functionality changes largely depend on the nature and structural integrity of the adsorbed proteins. The mechanical strength of natural hard tissues also results from the attachment of collagen and other proteins to their mineral parts. ${ }^{4}$ Bacterial attachment on the implant surface is also governed by the adsorbed protein-bacterial protein interactions. Because of such plethora of biophysical implications, protein-material interactions have acquired central importance in the field of biomaterials science.

Several physicochemical factors play important roles in protein adsorption phenomena. Protein adsorption kinetics is largely influenced by surface roughness, porosity, particle size, wettability, chemical groups on the substrate, surface defects, and vacancies. ${ }^{5}$ Adsorption probability also depends on the size of proteins and their amino acid sequences. ${ }^{5}$ In general, larger protein molecules show a greater potential to be adsorbed on

Received: September 10, 2020

Revised: December 18, 2020

Published: January 4, 2021 
material substrates. Because of higher diffusion rates, smaller molecules get adsorbed onto the surface first and are replaced by larger proteins later. ${ }^{6}$ Various factors, like $\mathrm{pH}$ and the presence of different ions, also influence the adsorption process. $^{5}$ In addition to these, the protein adsorption phenomena can also be modulated with the application of external stimuli (thermal, electric, and magnetic). ${ }^{7-9}$

In general, proteins change their conformation during the adsorption process, which depends on the substrate properties and the nature of the protein solution. ${ }^{10-12}$ These conformational changes have significant influence on the cell-material interactions. For instance, bacteria exhibit different affinities toward saliva proteins adsorbed on a material surface, compared to that in an aqueous solution. ${ }^{13}$ Also, denatured proteins cannot support cell adhesion on material substrates. It is worth noting that not all the conformational changes are beneficial for cell attachment. Hence, it is important to explore the conformational changes of proteins for a better understanding of the biological response of materials.

Different experimental techniques like atomic force microscopy (AFM), time-of-flight secondary mass spectroscopy (ToFSIMS), Fourier-transform infrared spectroscopy (FTIR), etc. have been used to probe the conformational changes of proteins during adsorption. ${ }^{14-17}$ However, probing protein material interactions at the single molecular level is extremely difficult by experimental means, and the nature of the interactions are not completely known to this date. ${ }^{18}$ Computational techniques come to rescue as they offer to explore the area beyond the experimental regime. Molecular dynamics (MD) simulation is arguably the most widely used approach to investigate the interactions between proteins and inorganic surfaces. Different types of surfaces, namely, pure metals, metal oxides, and carbon allotropes, have been modeled to study their affinity toward proteins. ${ }^{19}$ Among them, hydroxyapatite (HA), being the hard tissue mineral, is of particular interest because of its proven biocompatibility in living systems. Between two stable crystallographic structures of $\mathrm{HA}$, the hexagonal structure is widely recognized because of its presence in hard tissues. ${ }^{20}$ Hexagonal HA exhibits a $\mathrm{P}_{3} / \mathrm{m}$ crystallographic symmetry with the following lattice parameters: $a=9.432 \AA$ and $c=6.881 \AA^{21}$

A number of computational studies are reported to understand the interactions between the HA surface and proteins/ peptides/amino acids. ${ }^{22-25}$ It is now evident that the adsorption of amino acids on the HA surface takes place via electrostatic forces and the formation of $\mathrm{Ca}-\mathrm{O}$ and $\mathrm{H}$-bonds. ${ }^{25,26}$ In case of zwitterionic amino acids, interactions take place via $\mathrm{Ca}-\mathrm{O}$ bond formation. Because of this fact, many amino acids tend to adsorb on the HA surface in their zwitterionic form. ${ }^{27}$ An oft-called example is the adsorption of Gly on the HA (010) surface. ${ }^{26}$ The formation of $\mathrm{Ca}-\mathrm{O}$ and $\mathrm{O}-\mathrm{Ca}-\mathrm{O}$ bonds through proton transfer from amino acid to $\mathrm{HA}$ was evident from DFT calculations. ${ }^{26}$ Similar adsorption phenomena were recorded in the case of Lys. ${ }^{22}$ Apart from these, electrostatic interactions among oppositely charged groups $\left(\mathrm{COO}^{-}\right.$and $\mathrm{Ca}^{2+}, \mathrm{NH}_{3}{ }^{+}$and $\left.\mathrm{PO}_{4}{ }^{3-}\right)$ also regulate the overall adsorption process. ${ }^{26}$ It is quite natural that similar kind of interaction has been observed between whole protein molecules and different HA crystal facets. The adsorption of bone morphogenic protein-2 (BMP2), for example, has been found to be mediated by the electrostatic interactions between $-\mathrm{COO}^{-}$and $\mathrm{Ca}^{2+} \cdot{ }^{28,29}$ Water-bridged $\mathrm{H}$-bonds were present between the protein and the material surface as well. ${ }^{28}$ The adsorption mechanism of BMP-7, on the other side, was found to be dominated by the electrostatic interactions between $\mathrm{Ca}^{2+}$ and $-\mathrm{COO}^{-}$, together with the $\mathrm{H}$-bond formation between $\mathrm{NH}_{3}{ }^{+}$and $\mathrm{PO}_{4}{ }^{3-}{ }^{30}$

Another important protein is fibronectin (FN), which is an extracellular matrix protein having a molecular weight of $\sim 440$ $\mathrm{kDa}^{31}$ Three types of FN repeats (FNI, FNII, and FNIII) are found in each monomer of this dimer protein. ${ }^{31}$ Almost $90 \%$ of the FN sequence is composed of 12 FNI repeats, 2 FNII repeats, and 15-17 FNIII repeats. ${ }^{31}$ The adsorption study of the FNIII $_{10}$ module on the HA (001) surface established the electrostatic interaction force to be the governing factor. ${ }^{32}$ In case of the FNIII $_{7-10}$ module, two stages of adsorption, namely, weak adsorption and strong adsorption, were recorded. ${ }^{33}$ While the weak adsorption was driven by the VDW interaction energy, Coulombic interactions influenced the strong adsorption phase. ${ }^{33}$ The nature of interactions between different proteins like osteopontin (OPN), bone sialoprotein (BSP), and leucinerich amelogenin protein (LRAP) with the HA surface has also been investigated. ${ }^{34-36}$ More recently, the effects of dopant ions and other molecules on protein adsorption on the HA surface have been investigated. ${ }^{37,38}$

Despite these existing reports, the effects of external stimuli (electric or magnetic) on protein adsorption on the HA surface remains unexplored. Among various kinds of stimuli, the effect of external electric field on cell-functionality modulation has been recently investigated in some of our previous studies. ${ }^{39-41}$ Although the beneficial effect of electric field stimulation on bone cell growth and bone fracture healing is relatively well established, little is known about the underlying mechanisms. ${ }^{42}$ It is worth noting that all these phenomena are mediated by the protein-bioapatite (idealized as HA) interactions. ${ }^{43}$ Although the influence of an external electric field on protein adsorption has been reported in the context of $\mathrm{Si}$, carbon, and selfassembled monolayer surfaces, there is hardly any study on the effects of an electric field on protein-HA interactions. ${ }^{44-47}$

Against this background, the present study aims to bridge this gap by exploring the effect of electric field stimulation on protein-HA interactions, as a primary step to develop a better comprehension of the role of an electric field on cell-material interactions and different biological processes like fracture healing. The 10th type III module of FN and the HA (001) surface has been chosen as the model protein and surface, respectively. The HA (001) surface is one of the most explored surfaces, both experimentally and computationally. ${ }^{48,49}$ It has been observed that during the biomineralization of hard tooth tissues (dentine and enamel), crystal growth occurs overall at the (001) plane, that is, along the $c$-axis. ${ }^{50}$ It is clear that because HA platelets are elongated along the $c$ direction in bones and during the biomineralization of tooth hard tissues, the $\{100\}$ crystal planes play an important role in the interaction with molecules. ${ }^{50}$ Because of these reasons, one of the surfaces belonging to the $\{100\}$ family, that is, the HA (001) surface, has been used in the present study.

In this study, the external electric field-dependent protein conformational changes have been quantitatively probed using the all-atom molecular dynamics simulation method. The changes in the dipole moment orientation, hydrogen bond formation, and interaction energies with the strength and direction of the electric field have been assessed. In summary, the present study establishes a quantitative perspective on the mostly unexplored phenomena of the adsorption of FN on HA. 

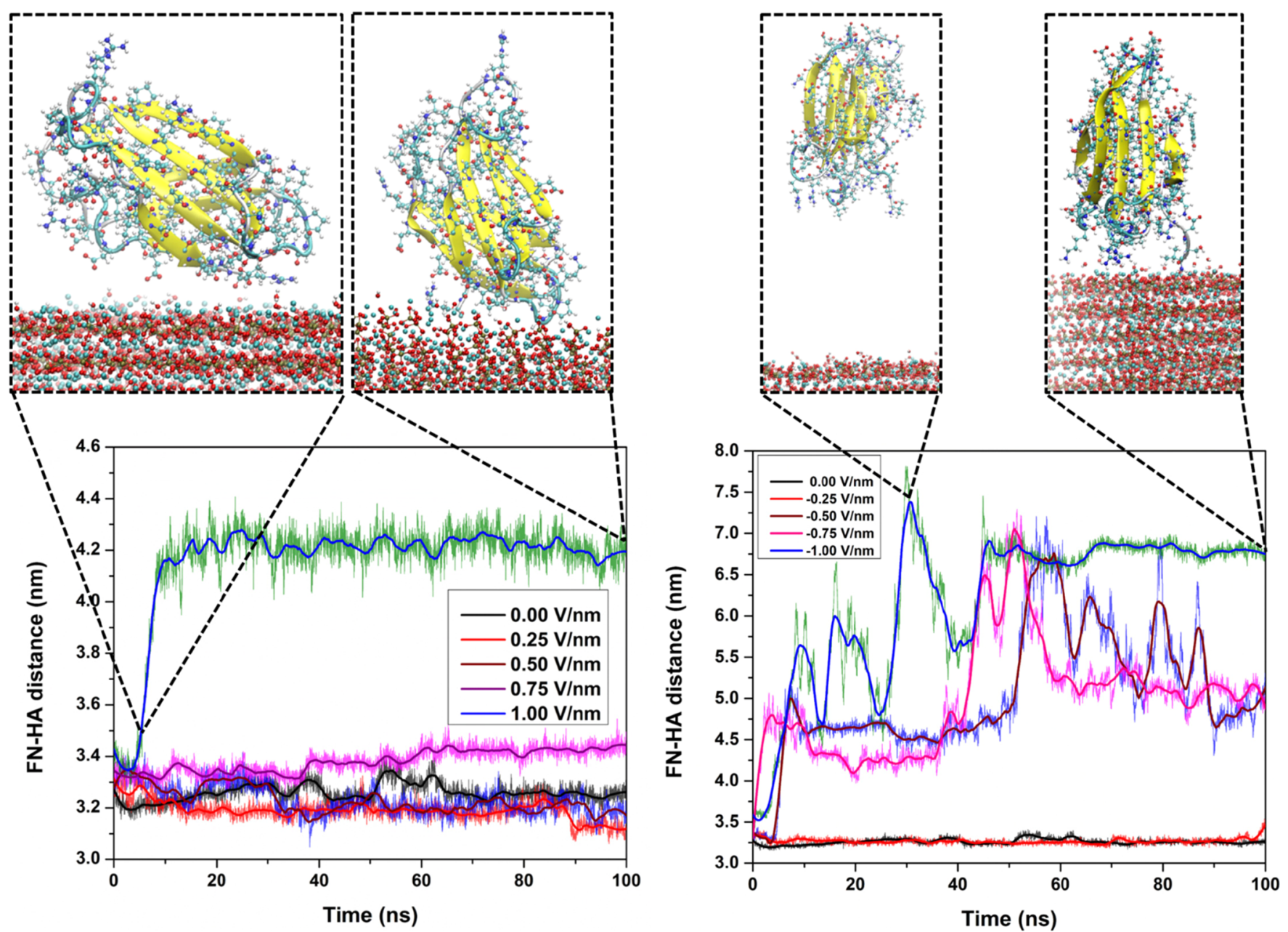

Figure 1. Electric field application and its directionality influence the adsorption kinetics. MD results to illustrate the temporal evolution of distance between the COM of FN and that of the HA substrate. The larger distance indicates that the high field strength does not favor FN adsorption on the HA surface. In the graphs, the legend color codes represent the thick lines (generated using a Savitzky-Golay filter). The snapshots of trajectory at different time points have been shown for $\pm 1.00 \mathrm{~V} / \mathrm{nm}$. Water molecules are not shown, for clarity. The secondary structure of FN has been represented with a color code: yellow: $\beta$ sheet, pale blue: turns, white: other residues. FN and HA have been presented using the CPK coloring scheme with a color code: red: $\mathrm{O}$, pale blue: $\mathrm{C}(\mathrm{FN})$ and $\mathrm{Ca}(\mathrm{HA})$, white: $\mathrm{H}$, blue: $\mathrm{N}$, and golden yellow: $\mathrm{P}$.

\section{SIMULATION DETAILS}

The structure of the model protein, $\mathrm{FN}-\mathrm{III}_{10}$, was taken from the PDB database (code: 1TTF). FN-III 10 consists of 94 residues with a molecular weight of $9.92 \mathrm{kDa}$. It has net zero charge, together with the total dipole moment of $245.7 \mathrm{D}$. This module is of particular interest because of the presence of an integrin binding site (RGD loop) in it. $^{32}$ The rectangular HA slab structure was obtained from the database of the INTERFACE force field (https://bionanostructures.com/interface-md/). This rectangular unit cell of HA features the following lattice parameters: $a=9.4170 \AA, b=16.3107 \AA, c=6.8750 \AA$, and $\alpha=\beta$ $=\gamma=90^{\circ}$.

A HA slab of $7.5336 \times 9.7864 \times 4.7500 \mathrm{~nm}^{3}$ was built and used as a biomaterials substrate in the MD simulation. A periodic box of size $7.5336 \times 9.7864 \times 14.04 \mathrm{~nm}^{3}$ was solvated with TIP3P water. The system contains 25,965 water molecules and an overall 96,715 atoms. Initially, the protein molecule was placed $5 \AA$ above the HA (001) facet, and the preferred adsorption orientation of the protein was chosen from the previous report. ${ }^{33}$ Energy minimization of the whole system was carried out in 10,000 steps by means of the steepest descent algorithm to remove close contacts between molecules. Then, the system was heated to $300 \mathrm{~K}$ in $1 \mathrm{~ns}$. A modified Berendsen thermostat $^{51}$ was employed for temperature coupling (at 300 $\mathrm{K}$ ), with a coupling constant of 0.1 ps. Additional $2 \mathrm{~ns}$ equilibration MD was carried out in an NPT ensemble with a reference pressure of 1.0 bar. Parrinello-Rahman barostat ${ }^{52}$ was used for pressure coupling with a coupling constant of 0.5 ps. Position restraints were applied on the proteins and HA slab during both the equilibration stages in the NVT and NPT ensembles. The coupling constant of the barostat was increased to $2.0 \mathrm{ps}$ during production runs. The integration time step of 2 fs was chosen throughout the simulation. External electric fields of up to $1.00 \mathrm{~V} / \mathrm{nm}$ were applied parallel and antiparallel to the z-axis. Throughout this article, positive and negative electric fields correspond to the electric field applied parallel and antiparallel to the z-direction, respectively. Position restraints on the HA surface were applied during production run only. Data from $100 \mathrm{~ns} \mathrm{MD}$ runs were collected for different kinds of analysis. All MD simulations were carried out in GROMACS 5.1.4 software, ${ }^{53}$ using the AMBER99SB ILDN force field. ${ }^{54}$ Force-field parameters for HA were taken from the INTERFACE force field. ${ }^{55}$ Necessary changes $(\mathrm{Kcal} / \mathrm{mol}$ to $\mathrm{kJ} / \mathrm{mol}$ and $\AA$ to $\left.\mathrm{nm} ; \sigma_{\mathrm{GROMACS}}=\left(\sigma_{\mathrm{INTERFACE}}\right) / 2^{1 / 6}\right)$ were performed to 

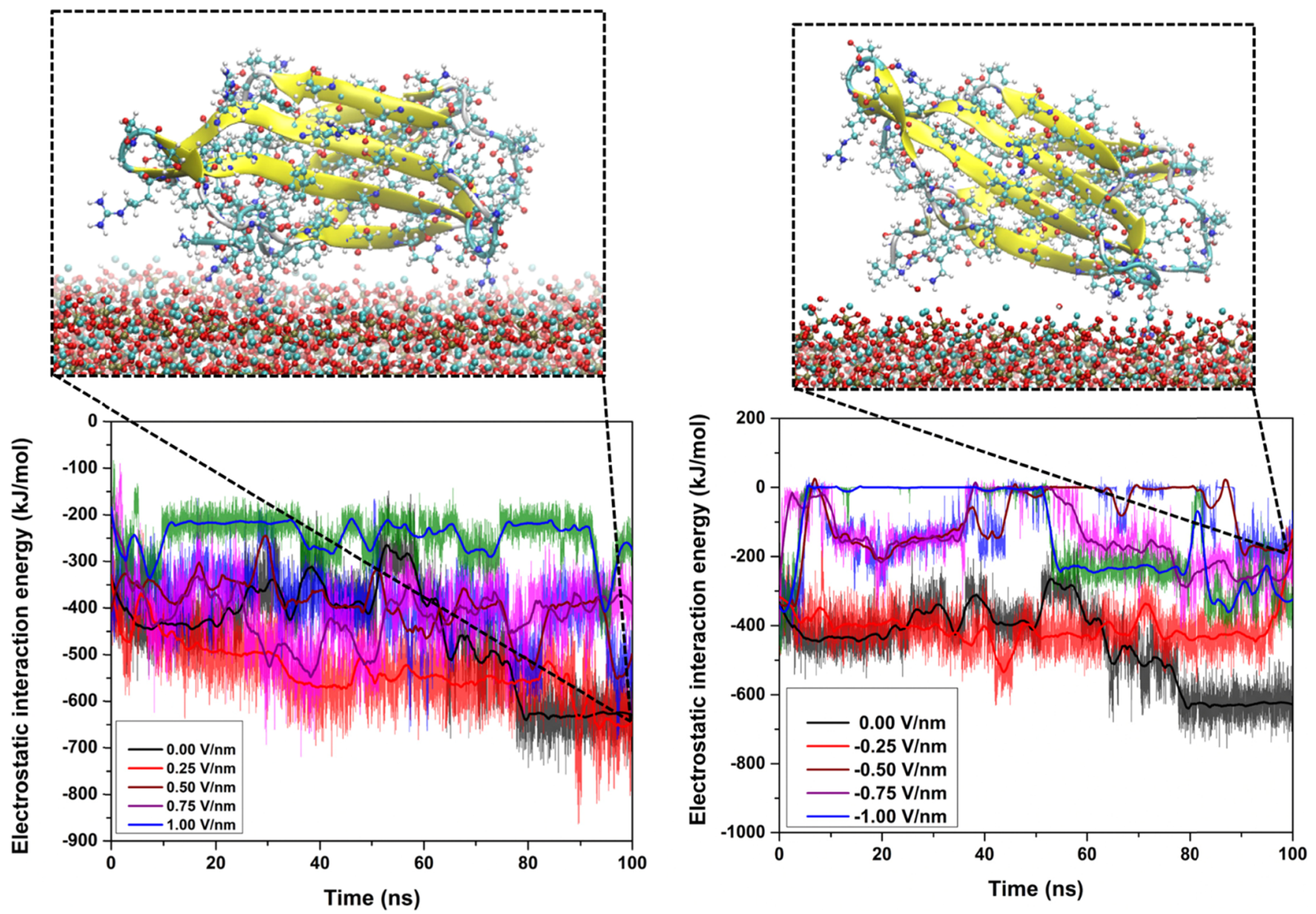

Figure 2. FN-HA interaction is dominated by the long-range Coulombic interaction. Time evolution of the electrostatic interaction energy between the FN-HA for different electric field strengths. Interaction strength generally reduces at a high field strength, which represents a weakly adsorbed state of FN. In the graphs, the legend color codes represent the thick lines (generated using a Savitzky-Golay filter). Final configurations (at $100 \mathrm{ns)}$ have been shown for $\pm 0.25 \mathrm{~V} / \mathrm{nm}$. Water molecules are not shown, for clarity. The secondary structure of FN has been represented with a color code: yellow: $\beta$ sheet, pale blue: turns, white: other residues. FN and HA have also been presented using the CPK coloring scheme with a color code: red: $\mathrm{O}$, pale blue: $\mathrm{C}(\mathrm{FN})$ and $\mathrm{Ca}(\mathrm{HA})$, white: $\mathrm{H}$, blue: $\mathrm{N}$, and golden yellow: $\mathrm{P}$.

make the INTERFACE force field parameters compatible with GROMACS. LINCS algorithm ${ }^{56}$ was employed for bond constraints (all bonds). Particle Mesh Ewald (PME) was used to calculate the long-range electrostatic interaction. ${ }^{57}$ A cutoff distance of $1.0 \mathrm{~nm}$ was adopted for short-range nonbonded interactions. The calculations of interaction energy, root-meansquare deviation (RMSD), dipole moment, hydrogen bonds, and protein surface distance were carried out with the help of different GROMACS modules. VMD 1.9.3 software $^{58}$ was used for visualization and salt bridge analysis. The cutoff used for the salt bridge and hydrogen bond analysis was set to 3.2 and $3.5 \AA$, respectively. The percentage of the occurrence of salt bridges was calculated from the relation $N_{\text {on }} / N_{\text {total }}$, where $N_{\text {on }}$ is the number of frames where the salt bridge was formed and $N_{\text {total }}$ is the total number of frames. ${ }^{59}$

\section{RESULTS}

We have employed all-atom MD simulation to probe into FN$\mathrm{HA}$ interactions with reference to the temporal evolution of interaction energy, dipole moment, hydrogen bonds, and other response parameters of the simulated system (Figure S1 in the Supporting Information). In the context of the cell-material interaction, the position of the RGD loop and its accessibility for the integrin protein is of particular interest. As iterated before, integrin, one of the major cell surface receptor proteins, interacts with FN through a specific amino acid sequence, and this interaction leads to the formation of the focal adhesion complex (Figure S1 in the Supporting Information). ${ }^{32}$ In this section, the adsorption behavior of $\mathrm{FN}$ is described with reference to the simulation results. The biophysical significance of the results will be described in the "Discussion" section.

3.1. FN-HA Interaction Energy. At the molecular level, the FN-HA interaction can be assessed by the temporal evolution of the distance of separation between the center of mass (COM) of the FN module and that of HA slab. This average distance is a signature of the occurrence of protein adsorption on the material surface. External electric fielddependent $\mathrm{COM}-\mathrm{COM}$ average distance has been plotted in Figure 1. For positive and low field strength (up to $0.50 \mathrm{~V} / \mathrm{nm}$ ), the average distance remains within the range of 3.1 to $3.3 \mathrm{~nm}$, throughout the simulation time, indicating the adsorbed state of protein (Figure 1). When the field was not applied, the distance between the protein and surface slightly increased from $3.1 \mathrm{~nm}$ at $\sim 3 \mathrm{~ns}$ up to $3.4 \mathrm{~nm}$ at $\sim 27 \mathrm{~ns}$. The oscillatory nature was recorded after this time up to $\sim 73 \mathrm{~ns}$, and a steady adsorbed state was obtained for the remainder of the simulation duration (Figure 1). In case of $0.25 \mathrm{~V} / \mathrm{nm}$, the protein got adsorbed on 
the HA surface around $20 \mathrm{~ns}$. A bump arose near $80 \mathrm{~ns}$, and the protein surface distance further reduced to $3.1 \mathrm{~nm}$ (Figure 1). At an increased field strength $(0.50 \mathrm{~V} / \mathrm{nm})$, a nearly constant protein surface distance of $3.2 \mathrm{~nm}$ was maintained from $\sim 54 \mathrm{~ns}$. For $0.75 \mathrm{~V} / \mathrm{nm}$, although the initial distance was around $3.35 \mathrm{~nm}$ up to $\sim 40$ ns of simulation, it started to increase after that. The distance reached $3.45 \mathrm{~nm}$ at $\sim 63 \mathrm{~ns}$. The increment of the COM-COM distance implies a comparatively weak adsorbed state. At $1.00 \mathrm{~V} / \mathrm{nm}$, the protein moved away from the surface within first $8 \mathrm{~ns}$ of simulation and maintained a nearly constant distance of $\sim 4.2 \mathrm{~nm}$ for the rest of the simulation time. In this case, the protein is loosely bound to the surface.

When field was applied antiparallel to the z-direction (negative z-direction), FN remained adsorbed on the surface for an applied field strength up to $-0.25 \mathrm{~V} / \mathrm{nm}$ (Figure 1). At this field strength, the protein surface distance was almost constant $(\sim 3.25 \mathrm{~nm})$ throughout the simulation time. With further increase in the field strength, the COM of the protein moved away from the surface and the adsorbed state became weaker (Figure 1). At a field strength beyond $-0.25 \mathrm{~V} / \mathrm{nm}, \mathrm{FN}$, at some time points, completely desorbed from the surface and again resorbed. The jumps in the distance profile at high electric fields represent the desorbed state of FN (Figure 1). For a field strength of $-1.00 \mathrm{~V} / \mathrm{nm}$, the desorbed state of FN remained for first $\sim 40$ ns of the simulation. The different behaviors of the $\mathrm{COM}-\mathrm{COM}$ distance at different field strengths indicate the influence of the external electric field on the adsorption phenomena of proteins on the material surface.

It is equally important to understand the modes of protein$\mathrm{HA}$ interaction. FN-HA interaction energies at different electric field strengths have been calculated using the GROMACS module and are represented in Figure 2. It is evident that the long-range attractive electrostatic interaction dominates FN adsorption on the HA surface. In the absence of the external field, the average electrostatic interaction between the protein and HA was around $-426.2 \pm 44.8 \mathrm{~kJ} / \mathrm{mol}$ for the first $21 \mathrm{~ns}$. An increase was recorded afterward up to $~ 54 \mathrm{~ns}$, followed by a sharp decrease. The interaction energy remained constant at $\sim-628.8 \pm 38.2 \mathrm{~kJ} / \mathrm{mol}$ from $80 \mathrm{~ns}$ onward. When the field strength was increased to $0.25 \mathrm{~V} / \mathrm{nm}$, a nearly steady state with an average interaction energy of $\sim-549.4 \pm 44.3 \mathrm{~kJ} /$ mol was reached at $\sim 50 \mathrm{~ns}$, and a further decrease was noted after 89 ns. A slight decrease in the interaction energy was observed at an electric field strength of $0.50 \mathrm{~V} / \mathrm{nm}$, whereas a nearly constant energy value of $\sim-403.6 \pm 53.1 \mathrm{~kJ} / \mathrm{mol}$ was recorded from $\sim 60 \mathrm{~ns}$ at a field strength of $0.75 \mathrm{~V} / \mathrm{nm}$. The Coulomb interaction energy between $\mathrm{FN}$ and HA fluctuates with a mean value of $\sim-245.2 \pm 5.8 \mathrm{~kJ} / \mathrm{mol}$ for $1.00 \mathrm{~V} / \mathrm{nm}$.

When the field direction is reversed, the situation was quite similar, only up to $-0.25 \mathrm{~V} / \mathrm{nm}$. The protein remained adsorbed on the surface throughout the simulation time (steady interaction energy). At a higher field, the interaction energy decreased further and sometimes approached zero during the investigated time window (Figure 2). These low interaction energies at high field correspond to a desorbed state of the protein.

The time-averaged electrostatic interaction energy is listed in Table 1. With the application of positive electric field, the average interaction energy first increased compared to that in the absence of the external field (Table 1 ). Beyond $+0.25 \mathrm{~V} / \mathrm{nm}$, it decreased nonmonotonously. On the other hand, the interaction energy started to decrease from the beginning, when the field direction was reversed. It increased at -0.75 and $-1.00 \mathrm{~V} /$
Table 1. Average Interaction Energy between FN and HA, as Calculated from the Trajectory Obtained from MD Simulation

$\begin{array}{ccc}\text { electric field strength }(\mathrm{V} / \mathrm{nm}) & \text { coulomb energy }(\mathrm{kJ} / \mathrm{mol}) & \text { error }(\mathrm{kJ} / \mathrm{mol}) \\ -1.00 & -133.5 & 53.0 \\ -0.75 & -139.1 & 38.0 \\ -0.50 & -92.3 & 30.0 \\ -0.25 & -408.2 & 9.8 \\ 0.00 & -454.3 & 50.0 \\ 0.25 & -533.0 & 25.0 \\ 0.50 & -392.3 & 19.0 \\ 0.75 & -413.2 & 18.0 \\ 1.00 & -245.2 & 5.8\end{array}$

$\mathrm{nm}$. Even at the same magnitude of field strength, the interaction energy was noted to be less, when the field was applied antiparallel to the $\mathrm{z}$-direction. The change in the attractive interaction energy establishes the fact that although FN adsorption was promoted at a low field strength (up to 0.25 $\mathrm{V} / \mathrm{nm}$ ), the application of high electric field does not favor protein adsorption on the HA surface.

Another important mode of interaction is mediated via the formation of hydrogen bonds between the material surface and $\mathrm{FN}$, which has been detailed in section S1 of the supplementary section.

For different electric field strengths, the number of contacts between HA and FN is presented in Figure 3, for last $10 \mathrm{~ns}$ of the total simulation time. The atom contact number was observed to reach the highest at $+0.25 \mathrm{~V} / \mathrm{nm}$ field strength (Figure 3 ). For a higher positive field strength, the number of contacts decreased, and the lowest values were recorded for $+1.00 \mathrm{~V} / \mathrm{nm}$. When the field direction was reversed, the number of contacts was found to be lesser for all field strengths, compared to that in the absence of any external field (Figure 3). In corroboration with the previously obtained results, the reduced number of $\mathrm{H}$-bonds and the number of contacts at a very strong field intensity represents the weakly adsorbed state of FN on HA.

3.2. Contact Residues and Secondary Structure. It is a well-known fact that in the course of the adsorption process, proteins change their native structures depending on the nature of the interaction with an inorganic surface. ${ }^{19}$ Only a few types of residues of any protein interact with the inorganic surface, depending on the nature of the surface bonds and the amino acids. The interacting residues of $\mathrm{FN}$, at different electric field strengths, together with their closest groups, are listed in Table 2. Arginine, aspartic acid, glycine, glutamic acid, etc. are the dominant residues that are found near the HA surface, and this result is in agreement with the existing literature. ${ }^{33}$ Among them, positively charged residues like arginine and lysine interact via the $\mathrm{NH}_{2} / \mathrm{NH}_{3}{ }^{+}$groups. Negatively charged residues (glutamic acid and aspartic acid) and neutral amino acids (glycine) were observed to interact with the HA surface through the $\mathrm{COO}^{-}$ groups. A notable observation is that beyond the field strength of $0.25 \mathrm{~V} / \mathrm{nm}$, the number of interacting residues decreased. Moreover, Arg93 residue ceased to interact with the HA surface at higher electric field strength. This effect was prominent when the field was applied antiparallel to the $\mathrm{z}$-axis. In a previous report, this residue (Arg93) was noted as the most strongly interacting residue with HA. ${ }^{33}$ Therefore, the withdrawal of Arg93 from the HA-FN interaction at higher field strengths reflects a weaker adsorbed state. Another notable observation is that the number of interacting residues decreased when the 

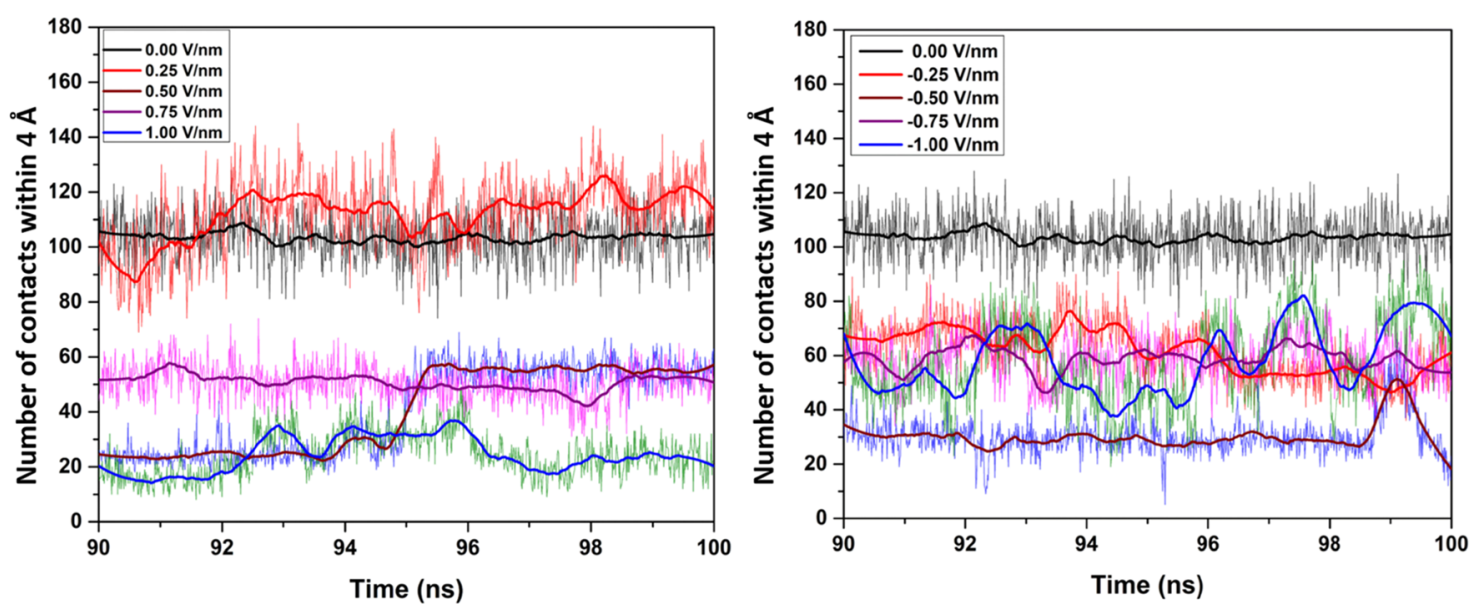

Figure 3. Larger number of atomic contacts is an indication of better adsorption. Time evolution of the number of contacts between FN and HA at different electric field strengths and directions. The highest number of contacts was formed in the case of $+0.25 \mathrm{~V} / \mathrm{nm}$, which can be correlated with the observed highest interaction strength between FN and HA at this field strength. A contact is defined when two atoms belonging to FN and HA are within a distance of $4 \AA$. In the graphs, the legend color codes represent the thick lines (generated using a Savitzky-Golay filter).

Table 2. Summary of Various Residues of FN Adsorbed on the HA Surface in the Presence of an Electric Field

\begin{tabular}{|c|c|}
\hline $\begin{array}{l}\text { electric field strength } \\
\qquad(\mathrm{V} / \mathrm{nm})\end{array}$ & anchoring residues (nearest group) \\
\hline-1.00 & $\operatorname{Val} 1\left(\mathrm{NH}_{3}^{+}\right)$, Ser2 \\
\hline-0.75 & $\operatorname{Arg} 78\left(\mathrm{NH}_{2}\right)$ \\
\hline-0.50 & $\operatorname{Arg} 78\left(\mathrm{NH}_{2}\right)$ \\
\hline-0.25 & $\begin{array}{l}\text { Asn42 }\left(\mathrm{NH}_{2}\right), \text { Gly40 }\left(\mathrm{COO}^{-}\right), \text {Asp67 }\left(\mathrm{COO}^{-}\right), \operatorname{Arg} 93 \\
\quad\left(\mathrm{NH}_{2}\right),\end{array}$ \\
\hline 0.00 & $\begin{array}{l}\text { Vall }\left(\mathrm{NH}_{3}^{+}\right), \mathrm{Glu} 9\left(\mathrm{COO}^{-}\right), \text {Asp7 }\left(\mathrm{COO}^{-}\right), \operatorname{Arg} 93 \\
\quad\left(\mathrm{NH}_{2}\right),\end{array}$ \\
\hline 0.25 & $\begin{array}{l}\operatorname{Arg} 6\left(\mathrm{NH}_{2}\right), \operatorname{Asp} 3\left(\mathrm{COO}^{-}\right), \operatorname{Asp} 7\left(\mathrm{COO}^{-}\right), \mathrm{Lys} 86 \\
\left(\mathrm{NH}_{3}^{+}\right), \operatorname{Arg} 93\left(\mathrm{NH}_{2}\right)\end{array}$ \\
\hline 0.50 & Asp7 $\left(\mathrm{COO}^{-}\right)$, Glu9 $\left(\mathrm{COO}^{-}\right), \operatorname{Arg} 93\left(\mathrm{NH}_{2}\right)$ \\
\hline 0.75 & $\operatorname{Arg} 93\left(\mathrm{NH}_{2}\right)$, Glu9 $\left(\mathrm{COO}^{-}\right)$, Val11 $\left(\mathrm{COO}^{-}\right)$, \\
\hline 1.00 & $\begin{array}{l}\text { Thr94 }\left(\mathrm{COO}^{-}\right) \text {, Gly65 }(\alpha-\mathrm{C}) \text {, Pro64 (part of side } \\
\text { chain) }\end{array}$ \\
\hline
\end{tabular}

electric field was applied along the negative $\mathrm{z}$ direction (Table 2). The change in the adsorbing residues with the change in the field strength and direction is a signature of the profound effects of external perturbations like electric field on adsorption kinetics.

The relevance of the RGD loop in the context of cell-material interactions has been mentioned before (Figure $\mathrm{S} 1$ in the Supporting Information). The position of the RGD loop at different field strengths and directions is shown in Figure 4. In the absence of an electric field, the RGD loop is exposed in the solution and is available for cell-material interactions (Figure 4). When the E-field was applied parallel to the positive $\mathrm{z}$-direction, the RGD loop remained exposed to the solvent for all field strengths (Figure 4). Up to $-0.25 \mathrm{~V} / \mathrm{nm}$, the RGD sequence was away from the HA surface (Figure 4). At the field strengths of -0.50 and $-0.75 \mathrm{~V} / \mathrm{nm}$, one of the residues (Arg78) of the RGD loop was adsorbed on the surface. At $-1.00 \mathrm{~V} / \mathrm{nm}$, the RGD loop remained very close to the surface (Figure 4). These configurations, therefore, will not promote cell-material interactions. Therefore, it can be commented that cell-material interactions can be modulated by changing the electric field strength and direction.

In order to obtain a qualitative idea about the conformational changes, the aligned initial and final structures are presented in Figure S4 in the Supporting Information. It can easily be seen that the structural changes took place mainly in the flexible loop region of the protein. Not much significant changes were noticed in the $\beta$-sheet structure, except at $-1.00 \mathrm{~V} / \mathrm{nm}$ (Figure S4 in the Supporting Information) because of its unique structural feature. There exists a distinguished pattern of the inter-residual $\mathrm{H}$-bonds in the $\beta$-sheet, which provides high structural stability. Hence, it is very difficult to alter this structure by means of any external stimuli.

In order to obtain a quantitative estimate of the structural changes, the percentage of the $\beta$-sheets was measured, and the number lies between 36 and 58\% (Figure 5). For the positivefield direction, the percentage of $\beta$-sheets increased up to 0.75 $\mathrm{V} / \mathrm{nm}$, and then, it decreased. For the negative direction, the increasing trend was observed up to $-0.50 \mathrm{~V} / \mathrm{nm}$ (Figure 5). Additionally, the RMSD of the backbone of the protein was calculated and is shown in Figure 6. It is worth noting that the RMSD first decreased with the application of the electric field $(+0.25 \mathrm{~V} / \mathrm{nm})$ and beyond $0.25 \mathrm{~V} / \mathrm{nm}$, it started to increase, irrespective of the field direction (Figure 6). In addition to this, at a high field strength, the RMSD was observed to be higher, when the field was applied antiparallel. This indicates toward the dependency of the conformational change of FN not only on the electric field strength but also on its direction.

The shape of proteins can be approximated by an ellipsoidal shape, which is defined by the principal moments of inertia and principal axes. ${ }^{60}$ The principal moments of inertia of FN and their ratios has been listed in Table 3. From the table, it can be easily seen that when an electric field was applied parallel to the $\mathrm{z}$-axis, $\mathrm{I}_{1}$ and $\mathrm{I}_{2}$ increased up to $0.50 \mathrm{~V} / \mathrm{nm}$ and then decreased. An opposite trend was noted for $I_{3}$. The ratio, $I_{1} / I_{3}$, also exhibited a similar pattern, $I_{1} / I_{2}$. When the field direction was reversed, the increasing trend was noticed only up to $-0.25 \mathrm{~V} /$ nm for $I_{1}, I_{2}$, and $I_{1} / I_{3}$. As before, $I_{3}$ exhibited an opposite trend. Various parameters calculated from the simulated trajectories to probe the structural change of FN clearly demonstrated that the electric field did not profoundly affect the structural integrity of the protein.

One of the ways to determine the structural integrity of proteins is to analyze the intramolecular salt bridge network, as it contributes significantly toward the thermostability of proteins. ${ }^{61}$ The number of salt bridges at different electric fields and the corresponding residues are presented in Table 4. When no 

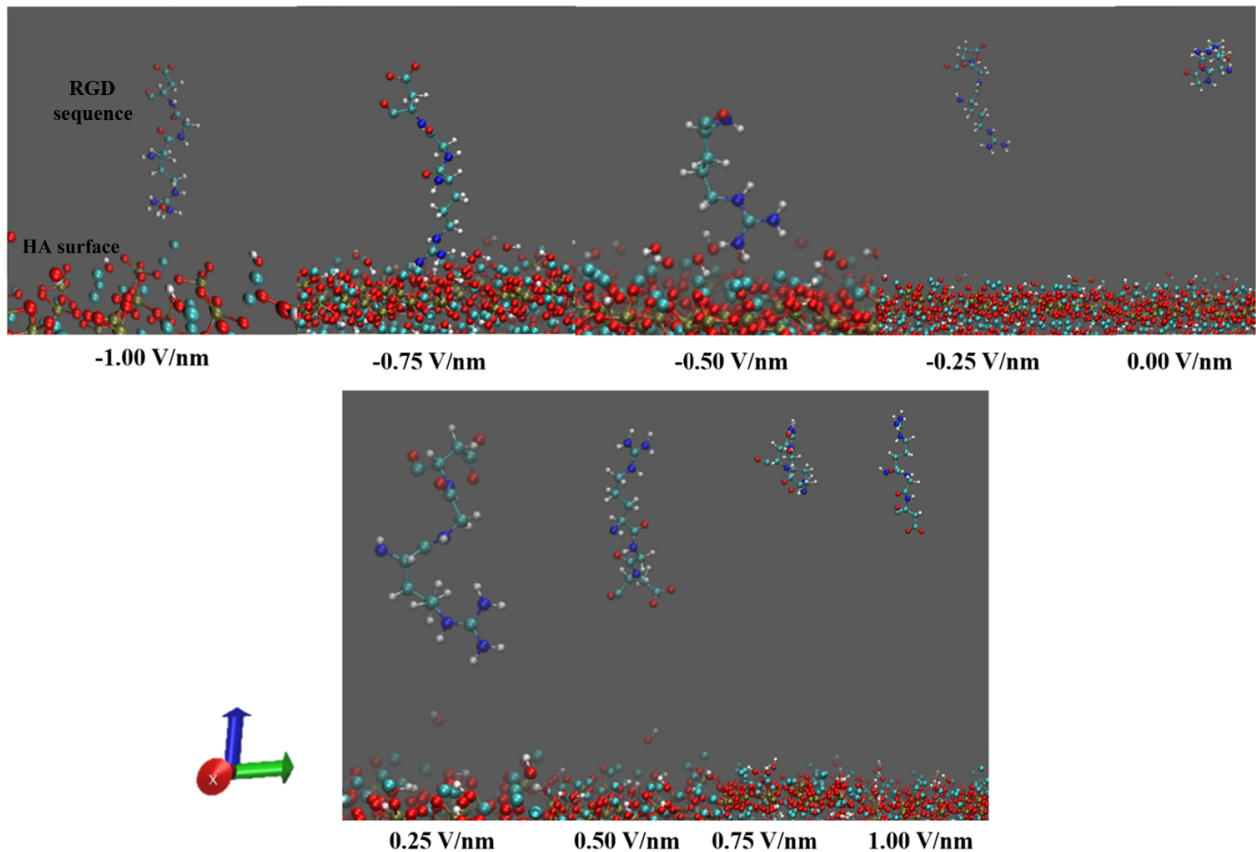

Figure 4. Accessibility of the RGD loop profoundly influences cell-material interactions. It is worth noting that the conformation with the RGD loop exposed to the solvent is suitable for cell-material interactions. The position of the RGD loop with respect to the HA surface after $100 \mathrm{~ns}$ of simulation at different electric fields. The RGD loop is not exposed to the solvent at the negative field direction. Hence, the application of a negative field is likely to hinder cell adhesion on the HA surface (Atomic color code: red: O, blue: N, pale blue: C (FN) and Ca (HA), white: H, and golden yellow: P. Axis color code: red: $x$, green: $y$, and blue: $z$ ).
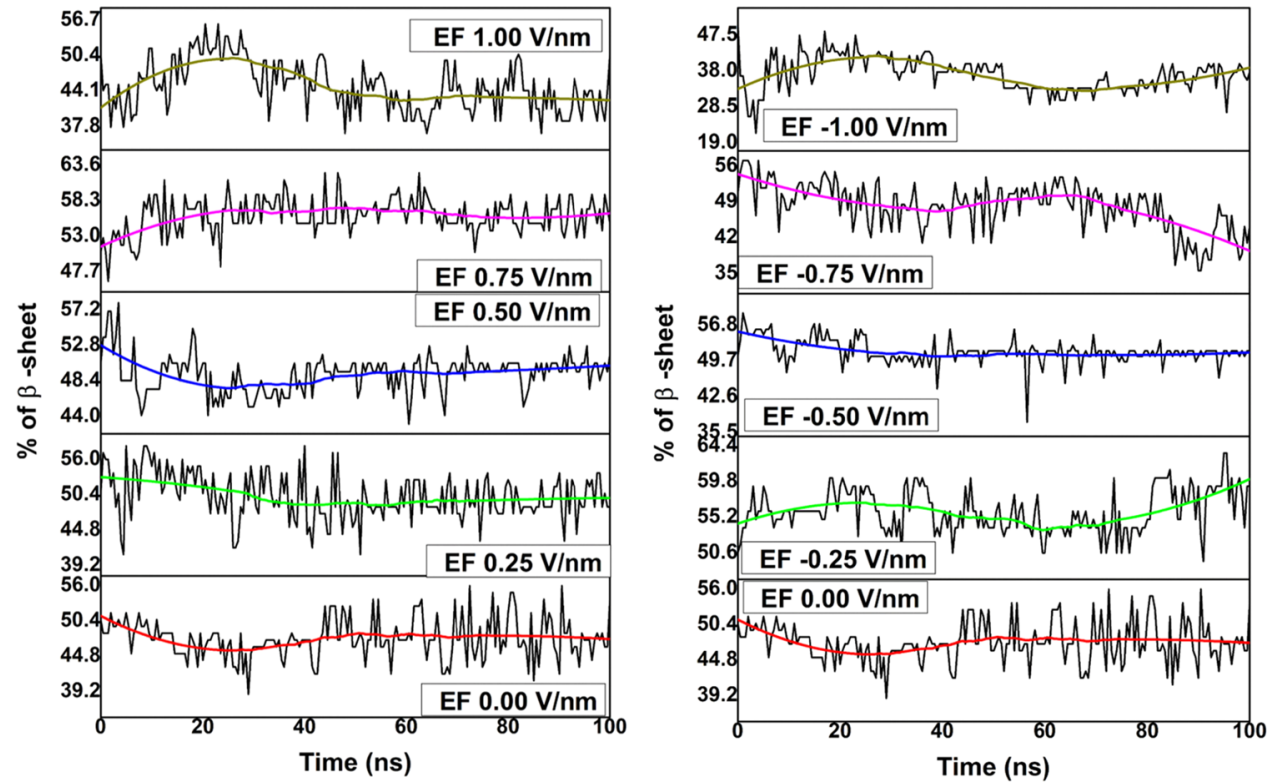

Figure 5. Electric field application impacts the structural integrity of FN (secondary structure). The percentage of $\beta$-sheet as a function of simulation time, at different electric field strengths and directions. The stable $\beta$-sheet structure was not affected significantly at a low field strength and in the positive direction. Therefore, the FN structure cannot be greatly altered using a low electric field intensity. Notable change occurred at $-1.00 \mathrm{~V} / \mathrm{nm}$. Smoothed thick lines were generated using a Savitzky-Golay filter.

external field was applied, Arg6 formed salt bridges with Asp3, Asp7, and Asp23, resulting in a salt bridge network. Another salt bridge network observed was Arg6-Asp3-Lys86 (Table 4). These two salt bridge nets together with other salt bridges are retained at a field strength of $+0.25 \mathrm{~V} / \mathrm{nm}$. Moreover, a new salt bridge was observed between Asp80 and Arg30, leading to a new salt bridge net consisting of Arg78-Asp80-Arg30. These networks were affected with the increase in the field strength.
At $0.50 \mathrm{~V} / \mathrm{nm}$, a new network, Asp80-Arg33-Glu47 was observed, and the salt bridge between Arg78-Asp80 and Asp3Lys86 was broken. More salt bridges were formed with the application of a stronger electric field (Table 4). The presence of Asp3-Lys86-Arg6 and Asp23-Arg6-Asp3 salt bridge networks was recorded in both 0.75 and $1.00 \mathrm{~V} / \mathrm{nm}$. Additional formation of salt bridges among Asp80-Arg33-Glu47 and Glu9-Arg93Asp67 was observed at $1.00 \mathrm{~V} / \mathrm{nm}$. 

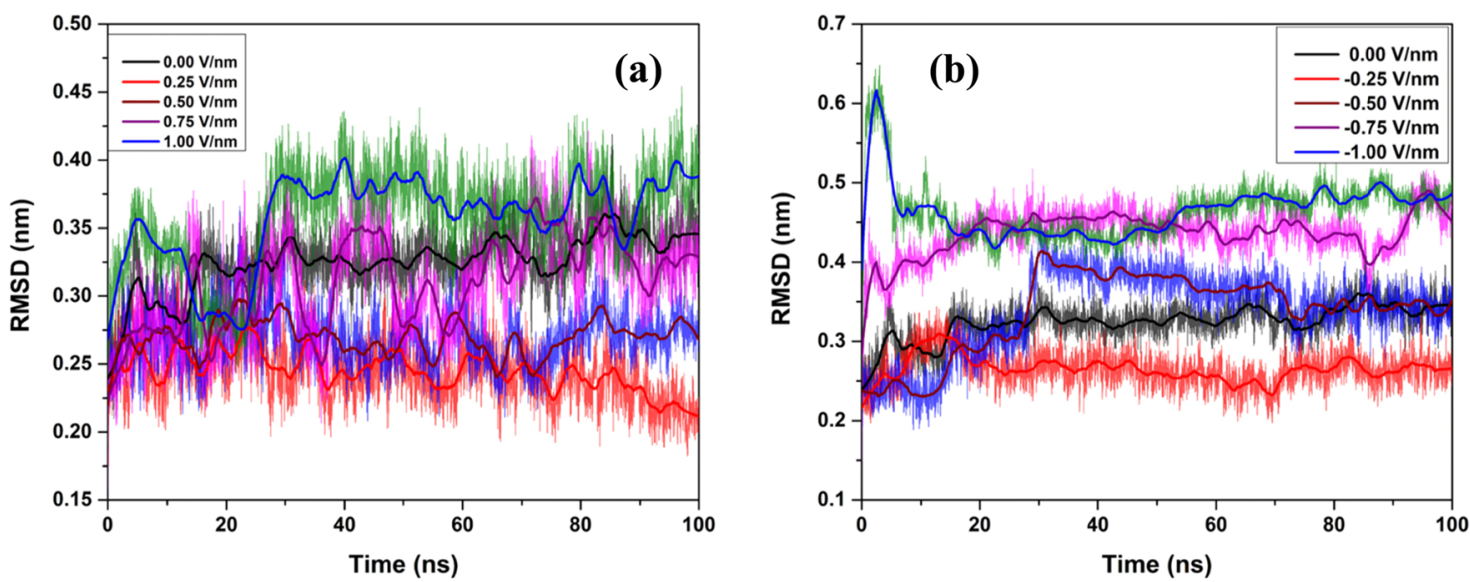

Figure 6. Structure of FN deviates from the initial structure within a narrow range in the presence of an electric field. RMSD of the FN-backbone as a function of (a) positive and (b) negative electric field strength. For both the positive and negative field strengths, RMSD first decreases and then increases with the magnitude of the electric field. The change in RMSD points to the fact that the electric field does not affect the FN structure in a drastic manner. Maximum changes occur in the flexible loop areas (see the article for details). In the graphs, the legend color codes represent the thick lines (generated using a Savitzky-Golay filter).

\section{Table 3. Principal Components of the Moment of Inertia of FN and Their Ratios}

\begin{tabular}{llllllc} 
electric field $(\mathrm{V} / \mathrm{nm})$ & $\mathrm{I}_{1}\left(\mathrm{amu} \AA^{2}\right)$ & $\mathrm{I}_{2}\left(\mathrm{amu} \AA^{2}\right)$ & $\mathrm{I}_{3}\left(\mathrm{amu} \AA^{2}\right)$ & $\mathrm{I}_{1} / \mathrm{I}_{2}$ & $\mathrm{I}_{1} / \mathrm{I}_{3}$ & $\mathrm{I}_{2} / \mathrm{I}_{3}$ \\
-1 & 2441743.5 & 2323944.25 & 1515877.125 & 1.050689 & 1.610779 & 1.533069 \\
-0.75 & 2192289 & 2115115.75 & 1412201.25 & 1.036487 & 1.552391 & 1.497744 \\
-0.5 & 2393993.25 & $2,299,658$ & 1109157.25 & 1.041021 & 2.158389 & 2.073338 \\
-0.25 & 2605389.75 & 2515012.5 & 1071420.25 & 1.035935 & 2.431716 & 2.347363 \\
0 & 2439327.25 & 2366321.75 & 1248351 & 1.030852 & 1.95404 & 1.895558 \\
0.25 & 2670087.5 & 2489611.5 & 1078987 & 1.072492 & 2.474624 & 2.30736 \\
0.5 & 2703543.5 & 2608899 & 1036026.063 & 1.036278 & 2.609532 & 2.518179 \\
0.75 & 2519970 & 2372511.5 & 1142999.5 & 1.062153 & 2.204699 & 2.075689 \\
1 & 2352267.75 & 2191058 & 1196919.625 & 1.073576 & 1.965268 & 1.830581 \\
no surface & 1696709 & 1588183.375 & 699086 & 1.068333 & 2.427039 & 2.2718 \\
\hline
\end{tabular}

Table 4. Salt Bridge-Forming Residues of FN at Different Electric Field Strengths

\begin{tabular}{cc}
$\begin{array}{c}\text { electric } \\
\text { field } \\
\text { strength } \\
\text { (V/nm) }\end{array}$ & \multicolumn{1}{c}{ salt bridge-forming residues } \\
-1.00 & Asp23-Arg6, Asp7-Arg93, Asp3-Lys86, Glu9-Arg93, Asp7- \\
& Lys54, Asp7-Arg6, Asp23-Lys54, Glu47-Arg33, Asp3-Arg6, \\
& Asp80-Arg33 \\
-0.75 & Asp80-Arg33, Asp23-Arg6, Asp7-Arg93, Glu9-Arg93, Asp7- \\
& Arg6, Asp23-Arg93, Asp3-Arg6, Glu47-Arg33, Asp3-Lys86, \\
& Glu9-Lys63, Asp23-Lys54, Asp7-Lys63 \\
-0.50 & Asp23-Arg6, Glu47-Arg33, Asp3-Lys86, Asp7-Arg6, Asp80- \\
& Arg30, Asp80-Arg78, Asp3-Arg6, Glu38-Lys63, Asp67-Arg93, \\
& Asp3-Arg78, Asp80-Arg33 \\
-0.25 & Asp23-Arg6, Glu47-Arg33, Asp3-Lys86, Asp67-Arg93, Asp7- \\
& Arg6, Asp80-Arg30, Glu38-Lys63, Asp3-Arg6 \\
0.00 & Asp23-Arg6, Asp80-Arg78, Asp3-Lys86, Glu47-Arg33, Asp67- \\
& Arg93, Asp7-Arg6, Glu38-Lys63, Asp3-Arg6 \\
0.25 & Asp80-Arg78, Glu47-Arg33, Asp3-Lys86, Asp80-Arg30, Asp7- \\
& Arg6, Glu38-Lys63, Asp67-Arg93, Asp3-Arg6, Asp23-Arg6 \\
0.50 & Asp80-Arg33, Glu47-Arg33, Asp80-Arg30, Asp67-Arg93, Asp23- \\
& Arg6, Glu38-Lys63, Asp3-Arg6 \\
0.75 & Asp80-Arg78, Asp23-Arg6, Asp3-Lys86, Glu47-Arg33, Asp67- \\
& Arg93, Asp7-Arg6, Glu38-Lys63, Asp3-Arg6 \\
1.00 & Asp80-Arg33, Asp3-Lys86, Glu47-Arg33, Glu9-Arg93, Asp67- \\
& Arg93, Asp80-Lys86, Asp23-Arg6, Glu38-Lys63, Asp3-Arg6 \\
\hline & \\
&
\end{tabular}

A noteworthy observation is that more salt bridges were formed in the presence of a strong electric field, when the field direction was reversed (Table 4). Salt bridge networks, formed in the presence of negative electric fields, were also noticed to be marginally different from those that appeared in the presence of the positive electric field. For example, Lys54 residue formed a salt bridge with Asp23 at $-0.75 \mathrm{~V} / \mathrm{nm}$ and with both Asp23 and Asp7 at $-1.00 \mathrm{~V} / \mathrm{nm}$. This particular residue did not take part in the salt bridge formation in the presence of a positive electric field. Asp7-Arg93-Glu9 is another such salt bridge net, which was formed only in association with an antiparallel electric field. The temporal evolution of these salt bridges is an interesting facet of this study to analyze the stability of the FN structure. The details of this particular aspect have been included in section S2 of the supplementary section.

3.3. Dipole Moment Distribution. The dipole moment of various system components plays an important role in the adsorption process. The dipole moment orientation of FN is shown in Figure 7a,b. The total dipole moment of protein after $100 \mathrm{~ns}$ of simulation and the corresponding angle with the $\mathrm{z}$-axis has been listed in Table 5. From Table 5, it is clear that the total dipole moment of the protein increased with an increase in the field strength, irrespective of the direction. Also, the dipoles tended to align themselves with the field direction. The zcomponent of the total dipole moment of FN is plotted in Figure $7 \mathrm{c}$,d. From the graphs, it can easily be noticed that although the magnitude of the z-component of the dipole moment increased with the increase in the field strength, the increase depended on the field direction (Figure $7 \mathrm{c}, \mathrm{d}$ ). An increase in the dipole moment took place within 100 ps of the simulation time (Figure 7). The other components ( $x$ and $y$ ) of the dipole moment 

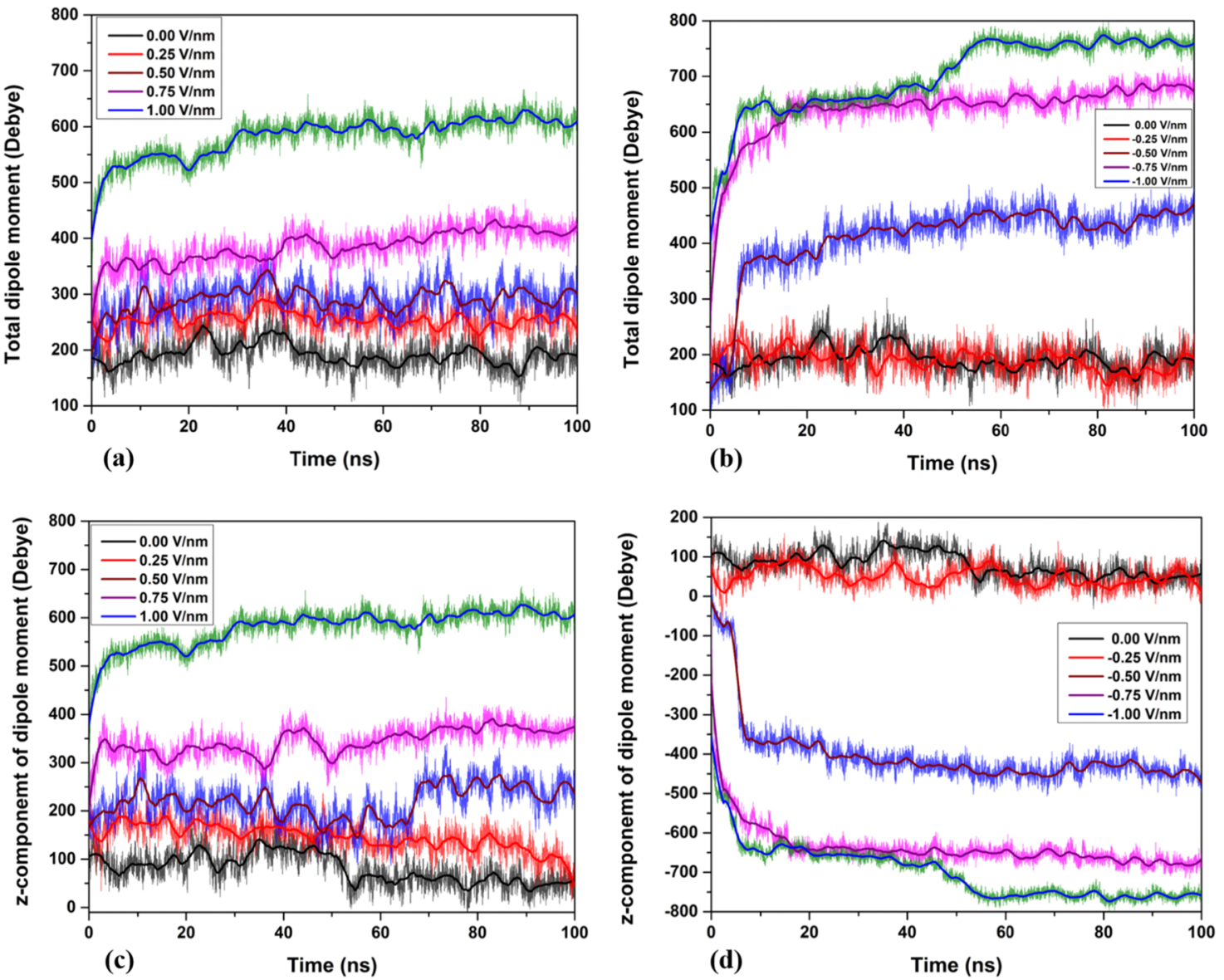

Figure 7. Dipole moment of protein changes with the magnitude and the direction of an external electric field. Time evolution of (a, b) total and (c, d) $z$-component of the dipole moment of protein at different electric fields. The change in the total dipole moment with the increase in the field strength can be corroborated with the rearrangement of the positions of local charges of FN. The recorded trend of the z-component of dipole moment indicates that the FN dipole moment vector orients itself along the field direction. In the graphs, the legend color codes represent the thick lines (generated using a Savitzky-Golay filter).

Table 5. Dipole Moment of FN at Different Electric Fields after $100 \mathrm{~ns}$ of Simulation and Their Orientation Relative to the $\mathrm{z}$-Axis

\begin{tabular}{cccc}
$\begin{array}{c}\text { electric field strength }(\mathrm{V} / \\
\mathrm{nm})\end{array}$ & $\begin{array}{c}\text { dipole moment } \\
\text { (debye) }\end{array}$ & $\cos \theta$ & $\begin{array}{c}\text { angle } \\
\text { (degree) }\end{array}$ \\
-1.00 & 751.9 & -1.00 & 180.0 \\
-0.75 & 689.7 & -1.00 & 180.0 \\
-0.50 & 500.1 & -0.99 & 171.9 \\
-0.25 & 233.2 & -0.05 & 92.9 \\
0.00 & 225.8 & 0.22 & 77.3 \\
0.25 & 228.6 & 0.32 & 71.3 \\
0.50 & 302.4 & 0.83 & 33.9 \\
0.75 & 418.1 & 0.91 & 24.5 \\
1.00 & 589.0 & 1.00 & 0.0 \\
\hline
\end{tabular}

vector of FN did not exhibit much significant change (Figure S8 in the supplementary section).

The total dipole moment of HA after $100 \mathrm{~ns}$ simulation time is tabulated in Table 6. When the field was applied along the $\mathrm{z}$ direction, the magnitude of the total dipole moment of HA decreased first, and then, it began to increase (Table 6). On the other hand, a monotonous increasing trend was noted when the field direction was reversed. Moreover, like FN, the angle between the dipole moment and $\mathrm{z}$-axis decreased and increased monotonously with positive and negative electric fields,
Table 6. Dipole Moment of the Bulk HA Surface as a Function of Electric Field Strength and the Direction at a Time Point of $100 \mathrm{~ns}^{a}$

$\begin{array}{cccc}\begin{array}{c}\text { electric field strength (V/ } \\ \mathrm{nm})\end{array} & \begin{array}{c}\text { dipole moment } \\ \text { (debye) }\end{array} & \cos \theta & \begin{array}{c}\text { angle } \\ \text { (degree) }\end{array} \\ -1.00 & 17948.4 & -0.82 & 145.1 \\ -0.75 & 13847.4 & -0.92 & 156.9 \\ -0.50 & 12491.2 & -0.71 & 135.2 \\ -0.25 & 7947.8 & -0.64 & 129.8 \\ 0.00 & 8180.6 & -0.35 & 110.5 \\ 0.25 & 8191.3 & -0.08 & 94.6 \\ 0.50 & 8448.4 & 0.27 & 74.3 \\ 0.75 & 11123.2 & 0.66 & 48.7 \\ 1.00 & 18954.2 & 0.64 & 50.2\end{array}$

${ }^{a}$ Angle made by the dipole moment vector with the z-direction is also shown.

respectively (up to $0.75 \mathrm{~V} / \mathrm{nm}$ ). This trend implies that the dipole moment vector of HA was trying to align itself with the field direction, similar to the protein. The z-component of the dipole moment also followed a similar pattern (Figure S9 in the Supporting Information).

The electrical behavior of water was found to follow the similar trend, as depicted in Table 7. In this case, the angle between the dipole moment vector and the $\mathrm{z}$-axis decreased with 
Table 7. Dependency of Magnitude and the Orientation of the Total Dipole Moment Vector of Water on the Applied Electric field $^{a}$

$\begin{array}{cccc}\begin{array}{c}\text { electric field strength (V/ } \\ \mathrm{nm})\end{array} & \begin{array}{c}\text { dipole moment } \\ \text { (debye) }\end{array} & \cos \theta & \begin{array}{c}\text { angle } \\ \text { (degree) }\end{array} \\ -1.00 & 44271.0 & -1.00 & 180.0 \\ -0.75 & 38520.7 & -1.00 & 180.0 \\ -0.50 & 27663.0 & -1.00 & 180.0 \\ -0.25 & 16095.4 & -1.00 & 180.0 \\ 0.00 & 8059.5 & -1.00 & 180.0 \\ 0.25 & 1389.6 & 0.33 & 70.7 \\ 0.50 & 10228.0 & 1.00 & 0.0 \\ 0.75 & 26145.2 & 1.00 & 0.0 \\ 1.00 & 39221.9 & 1.00 & 0.0\end{array}$

${ }^{a}$ Value presented here is the instantaneous magnitude of the dipole moment vector at $100 \mathrm{~ns}$.

the increase in the field strength when applied parallel to the $\mathrm{z}$ direction (Table 7). On the other hand, the very same angle remained constant when the field direction was reversed. The magnitude of the dipole moment vector increased with the field strength, irrespective of the direction, except for $-0.25 \mathrm{~V} / \mathrm{nm}$ (Table 7). The z-component of the dipole moment exhibited a trend similar to that of FN/HA. It increased with the positive electric field and decreased with the negative field (Figure S10 in the Supporting Information).

The relative orientation of the dipole moment vector of $\mathrm{FN}$, $\mathrm{HA}$, and water has been presented in Figure 8, together with the principal axes of the moments of FN. With the increase in the field strength, all the dipole moment vectors tend to align themselves parallel to the field direction (Figure 8). The interactions among the dipole moment vectors of protein, HA, and water act as a major driving force of the protein adsorption process, as described in the next section.
3.4. Solvation Shell around FN. The hydration shell around the protein and the surface plays an important role in adsorption phenomena. To obtain an insight into the water structure surrounding the protein, we have also calculated the solvent radial distribution function using gmx tools. Figure S11 in the supplementary section presents the $g_{\mathrm{OC}}(r)$ of the water oxygen atoms surrounding the $\mathrm{C}_{\alpha}$ atom of a hydrophilic (Ser 53; Figure S11a,b) and a hydrophobic (Leu8; Figure S11c,d) residue at different electric field strengths. $r_{\max }$ was chosen as 2 $\mathrm{nm}$. For both the hydrophilic and hydrophobic residues, the radius of the first hydration shell was noted to be $\sim 0.4 \mathrm{~nm}$, irrespective of the field strength/direction (Figure S11 in the supplementary section). For the hydrophilic residue, the amplitude of the first peak of $g_{\mathrm{OC}}(r)$ appeared almost identical except for the high field strength, where it decreased. This observation implies the presence of a less amount of solvent surrounding the reference atom. On the other side, almost no change was recorded for the $g_{\mathrm{OC}}(r)$ of the hydrophobic residue, except at $-1.00 \mathrm{~V} / \mathrm{nm}$ (Figure S11 in the supplementary section). The significance of the obtained results are included in the Supporting Information (section S3 of the Supporting Information).

\section{DISCUSSION}

In this section, a correlation among different results described in the previous section will be discussed together with the detailed analysis of the underlying causes behind the recorded behavior of the protein. To achieve this, the established mechanisms of the protein-HA interaction will be iterated first.

4.1. Influence of the Electrostatic Interaction Energy on the Adsorption Kinetics. It is worth noting that not all the protein residues interact equally with the HA surface. Rather, there exists specific binding sites for this particular interaction. Phosphorylated amino acids constitute the first class of HA binding sites, and the second class is made up of $\gamma$ carboxyglutamic acid found in the mineral binding portion of
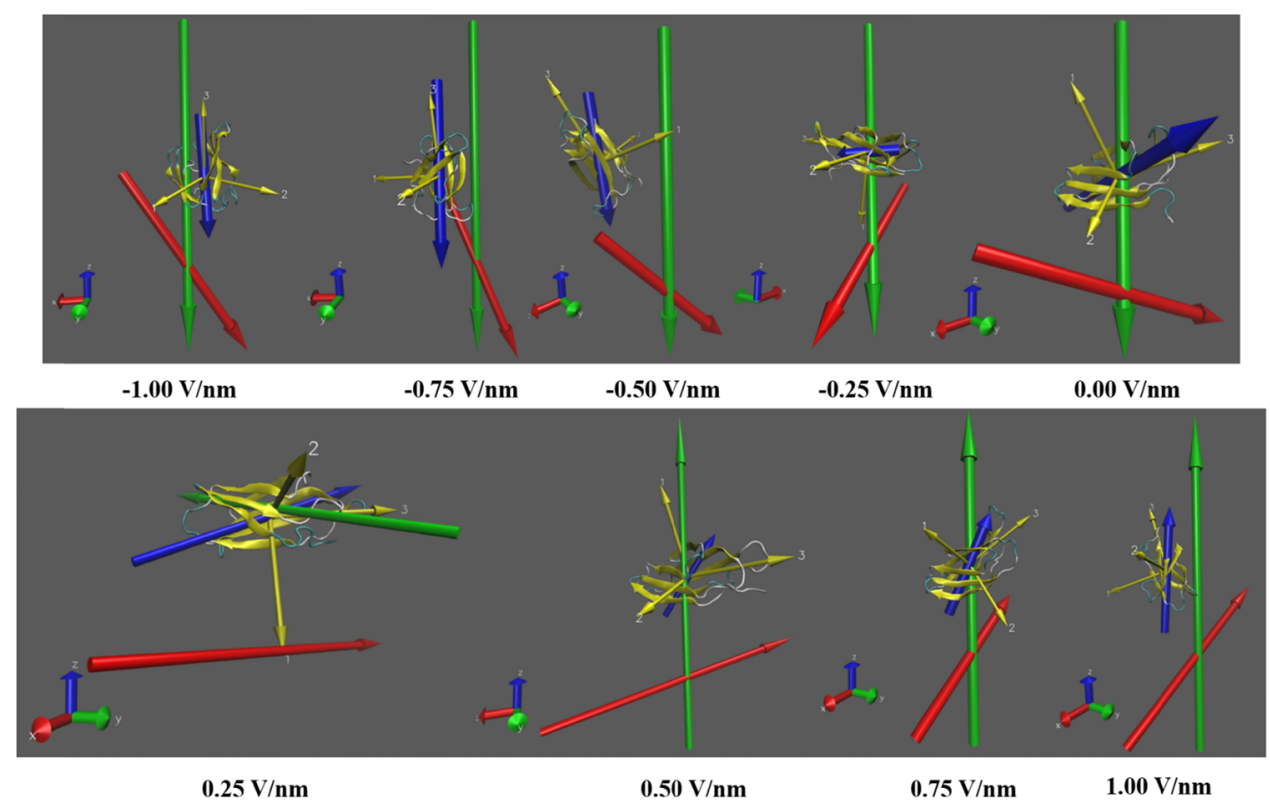

Figure 8. Dipole moment vector spontaneously aligns with the electric field direction. The dipole moment orientation of protein (blue), HA (red), and water (green) at different field strengths and directions. The principal axes of the momenta of FN are shown in yellow. At a high field strength, the dipole moment vectors of FN and water became parallel to each other, which increased the mutual repulsion between them and affected the adsorption process (axis color code: red: $\mathrm{x}$, green: $\mathrm{y}$, and blue: $\mathrm{z}$ ). The length of the arrows presenting the dipole moment vectors are not to scale. 
the bone Gla protein. ${ }^{62}$ While these two classes are composed of modified amino acids, a third class of HA binding site was composed of unmodified amino acids. ${ }^{62}$ The candidate for the third class of HA binding site is mainly the sequence of acidic amino acids. ${ }^{62}$ For example, the Asp or Glu-rich sections of proteins are found to bind with the HA crystal. ${ }^{62}$

In the present study, it has been observed that, mainly, the positively and negatively charged amino acids interacted with the HA surface (Table 2). Clearly, the electrostatic interaction among the oppositely charged groups of HA and FN mediated the adsorption phenomena. In some cases, neutral residues came closer to the surface during simulation, but their interaction with the surface was found to be weak compared to that of the charged residues (Figure S12 in the Supporting Information).

4.2. E-Field-Mediated Protein Conformational Changes and Stability. When a protein is exposed to the external electric field, two types of possible phenomena can take place to compensate the effect of the field. First, the rearrangement of the positions of the local atomic charges and the global conformational change of the whole protein. ${ }^{45}$ The combinational effects of these two phenomena result in the recorded behavior of FN on the HA surface. When the electric field strength was $0.25 \mathrm{~V} / \mathrm{nm}$, the first phenomena dominated, and the RMSD value was lower compared to that in the absence of the electric field. The local rearrangement of the positions of atomic charges brought more residues near the HA surface (Table 2). As a consequence, the number of hydrogen bonds between $\mathrm{HA}$ and $\mathrm{FN}$ increased together with the attractive electrostatic energy (Figure S2 in the supplementary section). Hence, a strong adsorbed state was observed along with reduced FN-HA distance (Figure 1).

When the field strength was increased to $0.50 \mathrm{~V} / \mathrm{nm}$, global structural changes of FN began to take place together with the local rearrangement. This reflected in the increased RMSD value and the reduced number of the interacting residues (Figure 6 and Table 2). Because of the presence of a smaller number of residues near the surface (Table 2), the number of hydrogen bonds and atomic contacts also decreased. The attractive interaction energy also decreased, and the FN-HA distance increased. As a result, the adsorbed state weakened. A similar trend followed with further increase in the field strength. Hence, one can conclude that beyond $0.25 \mathrm{~V} / \mathrm{nm}$, the application of electric field cannot support FN adsorption on the HA surface.

The scenario was slightly different when the field direction was reversed. At the field strength of $-0.25 \mathrm{~V} / \mathrm{nm}$, the number of interacting residues decreased compared to $0.00 \mathrm{~V} / \mathrm{nm}$, leading to a decrease in hydrogen bond formation and the number of contacts (Figure S2 in the supplementary section and Figure 3). There was also a slight decrease in the average attractive interaction energy (Table 1). This is probably due to the fact that the rearrangement of the positions of local atomic charges and global structural changes of FN happened in a different manner on the HA surface. The similar trend was observed at a field strength of $-0.50 \mathrm{~V} / \mathrm{nm}$. Here, the changes of the whole FN module were more prominent, as evident from the higher RMSD. The adsorption was also weak at this field strength. Only a few residues interact with the HA (001) surface (Table 2). As these residues are part of the RGD loop, the application of high electric field in an antiparallel direction is not suitable for the integrin-FN interaction. As cell-material interactions depend on the interactions between integrin and the RGD sequence of adsorbed FN, it will also not be facilitated when electric field is applied antiparallel to the z-direction.
When the field strength was further increased to $-0.75 \mathrm{~V} / \mathrm{nm}$, a behavior similar to that at $-0.50 \mathrm{~V} / \mathrm{nm}$ was observed. The interacting residue was the same as $-0.50 \mathrm{~V} / \mathrm{nm}$. The notable difference is that the attractive interaction energy, the number of contacts, and the frame average number of hydrogen bonds increased at $-0.75 \mathrm{~V} / \mathrm{nm}$ field strength (Figures 2 and 3 and Figure S3 in the Supporting Information). The underlying reason may be the different structural changes of FN under the influence of these two electric field strengths. Moreover, the main contribution toward the interaction energy and hydrogen bond formation came from the interacting Arg78 residue. It has been observed that between $\sim 54$ and $\sim 90 \mathrm{~ns}$, the COM-COM distance between Arg78 and HA was higher in case of $-0.50 \mathrm{~V} /$ $\mathrm{nm}$ compared to that at $-0.75 \mathrm{~V} / \mathrm{nm}$ (Figure S14a in the Supporting Information). Almost no hydrogen bond was formed between Arg78 and HA during this time period (Figure $\mathrm{S} 14 \mathrm{~b}$, Supporting Information), for $0.50 \mathrm{~V} / \mathrm{nm}$ field strength. This was reflected in the average number of $\mathrm{H}$-bonds. Because of a similar reason, Arg78 interacted with HA more strongly in case of $-0.75 \mathrm{~V} / \mathrm{nm}$ (Figure S14c in the supplementary section), and the total interaction energy increased (Figure 2).

At a field strength of $-1.00 \mathrm{~V} / \mathrm{nm}$, the protein-FN distance and RMSD increased significantly. Only two residues were close to the surface (Table 2), and the neutral residue interacted weakly with the HA surface (Figure S12c, Supporting Information). The major contribution toward the interaction energy and hydrogen bond formation came from the interaction of the Val1 residue with HA (Figure S13, Supporting Information). The number of absorbing residues increased at $-1.00 \mathrm{~V} / \mathrm{nm}$, and this was reflected by the slight increase in the number of contacts (Table 2 and Figure 3 ).

The prolate shape of FN was confirmed in the absence of the HA surface from the ratio of $I_{1} / I_{3}$ (Table 3 ). The same shape was retained in the presence of the surface and electric field as well. As the ratio of $I_{1} / I_{2}$ was found to be close to 1 , in all the cases, the ellipsoid shape of FN can crudely be approximated as a prolate spheroid. From the values of the principal components of moment, it can be concluded that FN first was elongated along the $c$-axis of the ellipsoid up to $+0.50 \mathrm{~V} / \mathrm{nm}$, and thereafter, it became shrunk. The same pattern was followed for the negative field direction as well. However, in this case, the decrease of $c$ axis started after $-0.25 \mathrm{~V} / \mathrm{nm}$ (Table 3 ).

There exists a clear dependency of the protein behavior on the field direction. The adsorption of FN was noted to be weaker when the field was applied antiparallel to the z-axis. Moreover, the RMSD was also found to be higher. The probable reasons can be the dipole-dipole interaction among FN, HA, and water. It is a well-known fact that the parallel dipoles experience a repulsive force and the antiparallel dipoles experience an attractive force. Among several factors, the force between the dipoles depends on the magnitude of the dipoles and on the cosine of the angle between them. ${ }^{63}$ In the present case, as the field strength was increased, the angle between the dipole moment vector of water and FN decreased irrespective of the field direction. As a consequence, the repulsive force between the dipoles increased, resulting in a weak adsorbed state at high electric field strength. Moreover, when the field was negative, the magnitude of dipole moments for both FN and water was higher compared to their counterparts with the positive electric field. This led to comparatively weaker adsorbed states of FN at negative electric fields.

The other important aspect of our study is to analyze the stability of the FN in the presence of the electric field. In the 
current study, it has been found that although more salt bridge networks were found to be formed at a high electric field strength (Table 4), the overall structural integrity was affected. The percentage of the occurrence of different salt bridges also strongly depends on the electric field strength, and the nature of dependency is specific to the residues taking part in the salt bridge formation (Figure S7 in the Supporting Information). In addition to this, the temporal stability of the salt bridges also reduced with the increase in the field strength, in general (Figure S7 in the Supporting Information). All of these phenomena can be attributed to the local rearrangement of the atomic positions of the protein to counteract the effect of the external field. The effect of the electric field on the O-N atomic pair distance in different salt bridges significantly varies because of the different locations of salt bridge-forming residues within the protein structure (Figure S6 in the Supporting Information). This results in the observed nondistinctive nature of the dependency of the occurrence frequency (percentage of occurrence) on the field strength and direction. At very high field strengths, the structure of FN significantly varies from its initial structure (as evident from higher RMSD), affecting the stability of most of the salt bridges, thus affecting the overall structural stability of FN.

\section{CONCLUSIONS}

In the present study, we have examined the physicochemical effect of electric field application on the FN adsorption on the HA (001) surface using the all-atom MD simulation technique. The following conclusions can be drawn from the present study.

a) The adsorption of FN on HA can be modulated by means of external electric field stimulation. While FN adsorption was favored at a low field strength (up to $+0.25 \mathrm{~V} / \mathrm{nm}$ ), the weakened adsorbed state was observed at a high field intensity $(>0.25 \mathrm{~V} / \mathrm{nm})$.

b) FN interacts with $\mathrm{HA}$ via electrostatic force. The charged residues contribute majorly to the $\mathrm{FN}-\mathrm{HA}$ interaction. The average attraction interaction energy first increased (up to $0.25 \mathrm{~V} / \mathrm{nm}$ ) and then decreased with the increase in the electric field (from $-454.3 \mathrm{~kJ} / \mathrm{mol}$ at $0.00 \mathrm{~V} / \mathrm{nm}$ to $-245.2 \mathrm{~kJ} / \mathrm{mol}$ at $1.00 \mathrm{~V} / \mathrm{nm})$.

c) The biophysical separation distance between $\mathrm{FN}$ and the HA surface increased from $\sim 32 \AA(0 \mathrm{~V} / \mathrm{nm})$ to $\sim 42 \AA$ ( 1 $\mathrm{V} / \mathrm{nm})$ and $\sim 66 \AA(-1 \mathrm{~V} / \mathrm{nm})$. Such a large separation distance indicates weak adsorbed state at a high field strength $(>0.25 \mathrm{~V} / \mathrm{nm})$.

d) The nonmonotonous behavior of RMSD has been explained in terms of two underlying phenomena: the spatial rearrangement of the positions of the local atomic charges and the global structural change of proteins.

e) An application of a high electric field $(>0.25 \mathrm{~V} / \mathrm{nm})$ towards the biomaterials surface (antiparallel to the $\mathrm{z}$ axis) does not facilitate cell-material interactions because of the proximity of the RGD loop to the HA surface.

f) The weakening effect of the external electric field on adsorption phenomena is more profound when the field is applied towards the HA surface (antiparallel to the z-axis). This can be implicated to the dipole-dipole interaction between $\mathrm{FN}$ and water.

In summary, the current study elucidates the effects of electric field on the structural changes and adsorption of FN on HA. This is of particular interest in the context of bioelectronics medicine where $\mathrm{FN}$ adsorption plays an important role in the interaction between the cellular and mineral parts of hard tissues in the presence of electrical stimuli. The findings of our study will provide primary guidelines to optimize an external stimulibased design of bioelectronics devices.

\section{ASSOCIATED CONTENT}

\section{Supporting Information}

The Supporting Information is available free of charge at https://pubs.acs.org/doi/10.1021/acs.jpcb.0c08255.

Salt bridge analysis of $\mathrm{FN}$; temporal evolution and time average of $\mathrm{H}$-bonds between $\mathrm{FN}$ and $\mathrm{HA}$; temporal evolution of the dipole moments of FN, HA, and water; solvation shell around $\mathrm{FN}$; interaction energy and COM$\mathrm{COM}$ distance between different residues and $\mathrm{HA}$; nonbonded interactions between $\mathrm{HA}$ and $\mathrm{FN}$; and results of additional $100 \mathrm{~ns}$ run for some selected electric field strength (PDF)

\section{AUTHOR INFORMATION}

\section{Corresponding Authors}

Bikramjit Basu - Materials Research Centre, Indian Institute of Science, Bangalore 560012, India; Center for Biosystems

Science and Engineering, Indian Institute of Science, Bangalore 560012, India; 이이.org/0000-0002-9154-5553;

Email: bikram@iisc.ac.in

Prabal K. Maiti - Center for Condensed Matter Theory, Department of Physics, Indian Institute of Science, Bangalore 560012, India; orcid.org/0000-0002-9956-1136;

Email: maiti@iisc.ac.in

\section{Authors}

Subhadip Basu - Materials Research Centre, Indian Institute of Science, Bangalore 560012, India

Biswajit Gorai - Center for Condensed Matter Theory, Department of Physics, Indian Institute of Science, Bangalore 560012, India; (1) orcid.org/0000-0003-1001-5567

Complete contact information is available at:

https://pubs.acs.org/10.1021/acs.jpcb.0c08255

\section{Notes}

The authors declare no competing financial interest.

\section{ACKNOWLEDGMENTS}

The authors acknowledge Sahasrat of Supercomputer Education and Research Centre (SERC) and the Thematic Unit of Excellence on Computational Materials Science (TUE-CMS) of Solid State and Structural Chemistry Unit (SSCU) at IISc, Bangalore, for providing access to the high-performance supercomputer facilities. One of the authors (BB) acknowledge DBT- National Bioscience award and Core Research Grant (CRG), Science and engineering research board (SERB), Department of Science and Technology (DST), Govt. of India for financial support. S.B. and B.B. also thank Prof. P. Balaram and Prof. M.K. Mathew, NCBS, Bangalore, for useful discussion. B.G. thanks Dr. D. S. Kothari Postdoctoral Fellowship scheme by University Grants Commission (UGC), India, for financial support.

\section{REFERENCES}

(1) Thrivikraman, G.; Boda, S. K.; Basu, B. Unraveling the Mechanistic Effects of Electric Field Stimulation towards Directing Stem Cell Fate and Function: A Tissue Engineering Perspective. Biomaterials 2018, 150, 60-86. 
(2) Chandorkar, Y.; Ravikumar, K.; Basu, B. The Foreign Body Response Demystified. ACS Biomater. Sci. Eng. 2018, 5, 19-44.

(3) Basu, B. Biomaterials for Musculoskeletal Regeneration; Springer, 2017.

(4) Viguet-Carrin, S.; Garnero, P.; Delmas, P. D. The Role of Collagen in Bone Strength. Osteoporos. Int. 2006, 17, 319-336.

(5) Wang, K.; Zhou, C.; Hong, Y.; Zhang, X. A Review of Protein Adsorption on Bioceramics. Interface Focus 2012, 2, 259-277.

(6) Vroman, L.; Adams, A. L. Findings with the Recording Ellipsometer Suggesting Rapid Exchange of Specific Plasma Proteins at Liquid/Solid Interfaces. Surf. Sci. 1969, 16, 438-446.

(7) Brusatori, M. A.; Tie, Y.; Van Tassel, P. R. Protein Adsorption Kinetics under an Applied Electric Field: An Optical Waveguide Lightmode Spectroscopy Study. Langmuir 2003, 19, 5089-5097.

(8) Szabelski, P.; Cavazzini, A.; Kaczmarski, K.; Liu, X.; Van Horn, J.; Guiochon, G. Experimental Studies of Pressure/Temperature Dependence of Protein Adsorption Equilibrium in Reversed-Phase HighPerformance Liquid Chromatography. J. Chromatogr. A 2002, 950, 4153.

(9) Nita, L. E.; Chiriac, A. P. Effect of Emulsion Polymerization and Magnetic Field on the Adsorption of Albumin on Poly(Methyl Methacrylate)-Based Biomaterial Surfaces. J. Mater. Sci. Mater. Med. 2010, 21, 2443-2452.

(10) Norde, W.; Giacomelli, C. E. BSA Structural Changes during Homomolecular Exchange between the Adsorbed and the Dissolved States. J. Biotechnol. 2000, 79, 259-268.

(11) Zhou, C.; Friedt, J.-M.; Angelova, A.; Choi, K.-H.; Laureyn, W.; Frederix, F.; Francis, L. A.; Campitelli, A.; Engelborghs, Y.; Borghs, G. Human Immunoglobulin Adsorption Investigated by Means of Quartz Crystal Microbalance Dissipation, Atomic Force Microscopy, Surface Acoustic Wave, and Surface Plasmon Resonance Techniques. Langmuir 2004, 20, 5870-5878.

(12) Dupont-Gillain, C. C.; Fauroux, C. M. J.; Gardner, D. C. J.; Leggett, G. J. Use of AFM to Probe the Adsorption Strength and Timedependent Changes of Albumin on Self-assembled Monolayers. J. Biomed. Mater. Res. A 2003, 67A, 548-558.

(13) Gibbons, R. J.; Hay, D. I.; Childs Iii, W. C.; Davis, G. Role of Cryptic Receptors (Cryptitopes) in Bacterial Adhesion to Oral Surfaces. Arch. Oral Biol. 1990, 35, S107-S114.

(14) Dufrêne, Y. F.; Marchal, T. G.; Rouxhet, P. G. Influence of Substratum Surface Properties on the Organization of Adsorbed Collagen Films: In Situ Characterization by Atomic Force Microscopy. Langmuir 1999, 15, 2871-2878.

(15) Zeng, H.; Chittur, K. K.; Lacefield, W. R. Analysis of Bovine Serum Albumin Adsorption on Calcium Phosphate and Titanium Surfaces. Biomaterials 1999, 20, 377-384.

(16) Bergkvist, M.; Carlsson, J.; Oscarsson, S. Surface-dependent Conformations of Human Plasma Fibronectin Adsorbed to Silica, Mica, and Hydrophobic Surfaces, Studied with Use of Atomic Force Microscopy. J. Biomed. Mater. Res. A 2003, 64A, 349-356.

(17) Henry, M.; Dupont-Gillain, C.; Bertrand, P. Conformation Change of Albumin Adsorbed on Polycarbonate Membranes as Revealed by ToF-SIMS. Langmuir 2003, 19, 6271-6276.

(18) Wang, Q.; Wang, M.; Wang, K.; Liu, Y.; Zhang, H.; Lu, X.; Zhang, $\mathrm{X}$. Computer Simulation of Biomolecule-Biomaterial Interactions at Surfaces and Interfaces. Biomed. Mater. 2015, 10, 32001.

(19) Ozboyaci, M.; Kokh, D. B.; Corni, S.; Wade, R. C. Modeling and Simulation of Protein-Surface Interactions: Achievements and Challenges. Q. Rev. Biophys. 2016, 49, No. e4.

(20) Langer, R. S.; Vacanti, J. P. Tissue Engineering: The Challenges Ahead. Sci. Am. 1999, 280, 86-89.

(21) Posner, A. S.; Perloff, A.; Diorio, A. F. Refinement of the Hydroxyapatite Structure. Acta Crystallogr. 1958, 11, 308-309.

(22) Lou, Z.; Zeng, Q.; Chu, X.; Yang, F.; He, D.; Yang, M.; Xiang, M.; Zhang, X.; Fan, H. First-Principles Study of the Adsorption of Lysine on Hydroxyapatite (1 00 ) Surface. Appl. Surf. Sci. 2012, 258, 4911-4916.

(23) Almora-Barrios, N.; Austen, K. F.; de Leeuw, N. H. Density Functional Theory Study of the Binding of Glycine, Proline, and
Hydroxyproline to the Hydroxyapatite (0001) and (0110) Surfaces. Langmuir 2009, 25, 5018-5025.

(24) Pan, H.; Tao, J.; Xu, X.; Tang, R. Adsorption Processes of Gly and Glu Amino Acids on Hydroxyapatite Surfaces at the Atomic Level. Langmuir 2007, 23, 8972-8981.

(25) Almora-Barrios, N.; de Leeuw, N. H. A Density Functional Theory Study of the Interaction of Collagen Peptides with Hydroxyapatite Surfaces. Langmuir 2010, 26, 14535-14542.

(26) Jimenez-Izal, E.; Chiatti, F.; Corno, M.; Rimola, A.; Ugliengo, P. Glycine Adsorption at Nonstoichiometric (010) Hydroxyapatite Surfaces: A B3LYP Study. J. Phys. Chem. C 2012, 116, 14561-14567.

(27) Basu, S.; Basu, B. Unravelling Doped Biphasic Calcium Phosphate: Synthesis to Application. ACS Appl. Bio Mater. 2019, 2, $5263-5297$.

(28) Dong, X.-L.; Qi, W.; Tao, W.; Ma, L.-Y.; Fu, C.-X. The Dynamic Behaviours of Protein BMP-2 on Hydroxyapatite Nanoparticles. Mol. Simul. 2011, 37, 1097-1104.

(29) Gu, H.; Xue, Z.; Wang, M.; Yang, M.; Wang, K.; Xu, D. Effect of Hydroxyapatite Surface on BMP-2 Biological Properties by Docking and Molecular Simulation Approaches. J. Phys. Chem. B 2019, 123, 3372-3382.

(30) Zhou, H.; Wu, T.; Dong, X.; Wang, Q.; Shen, J. Adsorption Mechanism of BMP-7 on Hydroxyapatite (001) Surfaces. Biochem. Biophys. Res. Commun. 2007, 361, 91-96.

(31) Pankov, R.; Yamada, K. M. Fibronectin at a Glance. J. Cell Sci. 2002, 115, 3861-3863.

(32) Shen, J.-W.; Wu, T.; Wang, Q.; Pan, H.-H. Molecular Simulation of Protein Adsorption and Desorption on Hydroxyapatite Surfaces. Biomaterials 2008, 29, 513-532.

(33) Liao, C.; Xie, Y.; Zhou, J. Computer Simulations of Fibronectin Adsorption on Hydroxyapatite Surfaces. RSC Adv. 2014, 4, 1575915769.

(34) Lai, Z. B.; Wang, M.; Yan, C.; Oloyede, A. Molecular Dynamics Simulation of Mechanical Behavior of Osteopontin-Hydroxyapatite Interfaces. J. Mech. Behav. Biomed. Mater. 2014, 36, 12-20.

(35) Yang, Y.; Cui, Q.; Sahai, N. How Does Bone Sialoprotein Promote the Nucleation of Hydroxyapatite? A Molecular Dynamics Study Using Model Peptides of Different Conformations. Langmuir 2010, 26, 9848-9859.

(36) Chen, X.; Wu, T.; Wang, Q.; Shen, J.-W. Shield Effect of Silicate on Adsorption of Proteins onto Silicon-Doped Hydroxyapatite (100) Surface. Biomaterials 2008, 29, 2423-2432.

(37) Alamdari, S.; Pfaendtner, J. Impact of Glutamate Carboxylation in the Adsorption of the $\alpha-1$ Domain of Osteocalcin to Hydroxyapatite and Titania. Mol. Syst. Des. Eng. 2020, 5, 620-631.

(38) Huang, B.; He, M.; Zhang, K.; Sun, S.; Lin, Z.; Chen, D.; Li, T.; Chen, X. Rotation-Induced Secondary Structure Losses and Bioactivity Changes of Bone Morphogenetic Protein-2 on Strontium-Substituted Hydroxyapatite Surfaces. Appl. Surf. Sci. 2020, 511, 145623.

(39) Thrivikraman, G.; Madras, G.; Basu, B. Intermittent Electrical Stimuli for Guidance of Human Mesenchymal Stem Cell Lineage Commitment towards Neural-like Cells on Electroconductive Substrates. Biomaterials 2014, 35, 6219-6235.

(40) Thrivikraman, G.; Madras, G.; Basu, B. Electrically Driven Intracellular and Extracellular Nanomanipulators Evoke Neurogenic/ Cardiomyogenic Differentiation in Human Mesenchymal Stem Cells. Biomaterials 2016, 77, 26-43.

(41) Ravikumar, K.; Kumaran, V.; Basu, B. Biophysical Implications of Maxwell Stress in Electric Field Stimulated Cellular Microenvironment on Biomaterial Substrates. Biomaterials 2019, 209, 54-66.

(42) Ryaby, J. T. Clinical Effects of Electromagnetic and Electric Fields on Fracture Healing. Clin. Orthop. Relat. Res. 1998, 355S, S205S215.

(43) Basu, B. Biomaterials Science and Tissue Engineering: Principles and Methods; Cambridge University Press, 2017.

(44) Xie, Y.; Gong, W.; Jin, J.; Zhao, Z.; Li, Z.; Zhou, J. Molecular Simulations of Lysozyme Adsorption on an Electrically Responsive Mixed Self-Assembled Monolayer. Appl. Surf. Sci. 2020, 506, 144962. 
(45) Xie, Y.; Liao, C.; Zhou, J. Effects of External Electric Fields on Lysozyme Adsorption by Molecular Dynamics Simulations. Biophys. Chem. 2013, 179, 26-34.

(46) Benavidez, T. E.; Torrente, D.; Marucho, M.; Garcia, C. D. Adsorption of Soft and Hard Proteins onto OTCEs under the Influence of an External Electric Field. Langmuir 2015, 31, 2455-2462.

(47) Mulheran, P. A.; Connell, D. J.; Kubiak-Ossowska, K. Steering Protein Adsorption at Charged Surfaces: Electric Fields and Ionic Screening. RSC Adv. 2016, 6, 73709-73716.

(48) Pan, H.; Tao, J.; Wu, T.; Tang, R. Molecular Simulation of Water Behaviors on Hydroxyapatite Crystal Faces. Chin. J. Inorg. Chem. 2006, 2, 1392.

(49) Duffy, D. M.; Harding, J. H. Growth of Polar Crystal Surfaces on Ionized Organic Substrates. Langmuir 2004, 20, 7637-7642.

(50) Corno, M.; Rimola, A.; Bolis, V.; Ugliengo, P. Hydroxyapatite as a Key Biomaterial: Quantum-Mechanical Simulation of Its Surfaces in Interaction with Biomolecules. Phys. Chem. Chem. Phys. 2010, 12, 6309-6329.

(51) Bussi, G.; Donadio, D.; Parrinello, M. Canonical Sampling through Velocity Rescaling. J. Chem. Phys. 2007, 126, 14101.

(52) Parrinello, M.; Rahman, A. Polymorphic Transitions in Single Crystals: A New Molecular Dynamics Method. J. Appl. Phys. 1981, 52, $7182-7190$

(53) Abraham, M. J.; Murtola, T.; Schulz, R.; Páll, S.; Smith, J. C.; Hess, B.; Lindahl, E. GROMACS: High Performance Molecular Simulations through Multi-Level Parallelism from Laptops to Supercomputers. SoftwareX 2015, 1-2, 19-25.

(54) Lindorff-Larsen, K.; Piana, S.; Palmo, K.; Maragakis, P.; Klepeis, J. L.; Dror, R. O.; Shaw, D. E. Improved Side-chain Torsion Potentials for the Amber Ff99SB Protein Force Field. Proteins Struct. Funct. Bioinforma. 2010, 78, 1950-1958.

(55) Lin, T.-J.; Heinz, H. Accurate Force Field Parameters and PH Resolved Surface Models for Hydroxyapatite to Understand Structure, Mechanics, Hydration, and Biological Interfaces. J. Phys. Chem. C 2016, 120, 4975-4992.

(56) Hess, B.; Bekker, H.; Berendsen, H. J. C.; Fraaije, J. G. E. M. LINCS: A Linear Constraint Solver for Molecular Simulations. J. Comput. Chem. 1997, 18, 1463-1472.

(57) Darden, T.; York, D.; Pedersen, L. Particle Mesh Ewald: An N. $\log (\mathrm{N})$ Method for Ewald Sums in Large Systems. J. Chem. Phys. 1993, 98, 10089-10092.

(58) Humphrey, W.; Dalke, A.; Schulten, K. VMD: Visual Molecular Dynamics. J. Mol. Graph. 1996, 14, 33-38.

(59) Desikan, R.; Maiti, P. K.; Ayappa, K. G. Predicting Interfacial Hot-Spot Residues That Stabilize Protein-Protein Interfaces in Oligomeric Membrane-Toxin Pores through Hydrogen Bonds and Salt Bridges. J. Biomol. Struct. Dyn. 2020, 1-15.

(60) Taylor, W. R.; Thornton, J. M. T.; Turnell, W. G. An Ellipsoidal Approximation of Protein Shape. J. Mol. Graph. 1983, 1, 30-38.

(61) de Bakker, P. I.; Hünenberger, P. H.; McCammon, J. A. Molecular Dynamics Simulations of the Hyperthermophilic Protein Sac7d from Sulfolobus Acidocaldarius: Contribution of Salt Bridges to Thermostability. J. Mol. Biol. 1999, 285, 1811-1830.

(62) Fujisawa, R.; Wada, Y.; Nodasaka, Y.; Kuboki, Y. Acidic Amino Acid-Rich Sequences as Binding Sites of Osteonectin to Hydroxyapatite Crystals. Biochim. Biophys. Acta (BBA)-Protein Struct. Mol. Enzymol. 1996, 1292, 53-60.

(63) Griffiths, D. J. Introduction to Electrodynamics; American Association of Physics Teachers, 2005. 